OHSTPY-HEP-T-97-18

hep-ph/9710357

October 14, 1997

\title{
Renormalons in Electromagnetic Annihilation Decays of Quarkonium
}

\author{
Eric Braaten and Yu-Qi Chen \\ Physics Department, Ohio State University, Columbus, Ohio 43210
}

\begin{abstract}
We study the large-order asymptotic behavior of the perturbation series for short-distance coefficients in the NRQCD factorization formulas for the decays $J / \psi \rightarrow e^{+} e^{-}$and $\eta_{c} \rightarrow \gamma \gamma$. The short-distance coefficients of the leading matrix elements are calculated to all orders in the large- $N_{f}$ limit. We find that there is a universal Borel resummable renormalon associated with the cancellation of the Coulomb singularity in the short-distance coefficients. We verify that the ambiguities in the short-distance coefficients from the first infrared renormalon are canceled by ambiguities in the nonperturbative NRQCD matrix elements that contribute through relative order $v^{2}$. Our results are used to estimate the coefficients of higher order radiative corrections in the decay rates for $J / \psi \rightarrow e^{+} e^{-}, \eta_{c} \rightarrow \gamma \gamma$, and $J / \psi \rightarrow \gamma \gamma \gamma$.
\end{abstract}




\section{INTRODUCTION}

The annihilation decay of heavy quarkonium involves several distance scales. The annihilation of the heavy-quark pair is a short-distance process occurring at length scales of order $1 / m$, where $m$ is the mass of the heavy quark. The probability that the heavy-quark pair will be close enough to annihilate is determined by the wavefunction of the heavy quarkonium, which is dominated by distances of order $1 / m v$ or larger, where $v$ is the typical relative velocity of the heavy quark. The nonrelativistic QCD (NRQCD) factorization formalism [1.2] is a systematic framework for separating the effects of the scale $m$ from effects of lower momentum scales. The annihilation decay rate is factored into short-distance coefficients and long-distance NRQCD matrix elements. The short-distance coefficients can be calculated using perturbative QCD as a power series in the strong coupling constant $\alpha_{s}(m)$ at the scale of the heavy quark mass. The matrix elements are nonperturbative, but scale in a definite way with $v$. The NRQCD factorization formalism therefore organizes the decay rate into a double expansion in powers of $\alpha_{s}$ and $v$.

In principle, the short-distance coefficient for each matrix element can be calculated to any desired order in $\alpha_{s}$ by matching the NRQCD effective theory with full QCD. One might hope that any desired precision could be obtained by carrying out the matching calculations to sufficiently high order in $\alpha_{s}$. However, large numbers may arise in high order calculations through the factorial growth of the coefficients in the perturbation series. A factorially growing series, when truncated at a high order, may produce a large error even if the coupling constant is small. This may limit the possibility of obtaining sufficiently precise results by performing the perturbative calculations to higher orders.

One can investigate the asymptotic behavior of a series $f\left(\alpha_{s}\right)$ by studying its Borel transform $f(t)$. A singularity in the Borel parameter $t$ is called a renormalon [3]. A renormalon gives rise to $n$ ! growth of the coefficients in the power series for $f\left(\alpha_{s}\right)$ so that its radius of convergence is 0 . If the renormalon is on the negative real axis, Borel resummation can be used to recover the function uniquely from the divergent series. If the renormalon is on the positive real axis, the sum of the divergent series is not unique and there is an ambiguity in the function $f\left(\alpha_{s}\right)$.

The renormalons that are responsible for the factorial growth of coefficients in the perturbation series for QCD have been extensively studied. Important applications include the operator product expansion for $e^{+} e^{-}$annihilation and $\tau$ decay [4] and heavy quark effective theory [8 11]. The large- $N_{f}$ limit of QCD has been developed by Beneke [5] into a useful tool for the explicit study of renormalons. In this paper, we apply this tool to the NRQCD factorization formulas for $S$-wave quarkonium states, focusing on electromagnetic annihilation decays.

The rest of this paper is organized as follows. In section II, we calculate the shortdistance coefficients for the electromagnetic annihilation decays $J / \psi \rightarrow e^{+} e^{-}$and $\eta_{c} \rightarrow$ $\gamma \gamma$ at tree level. We present a new method for calculating the tree-level short-distance coefficients which involves expanding nonlocal QCD matrix elements in terms of local QCD matrix elements, and then using a Foldy-Wouthuysen-Tani transformation to express them in terms of NRQCD matrix elements. In section III, we calculate the Borel transforms of the short-distance coefficients of the leading matrix elements in the large- $N_{f}$ limit. In section IV, we study the renormalons in the short-distance coefficients. We show that there 
is a universal Borel resummable renormalon related to the cancellation of the Coulomb singularity. We demonstrate that the ambiguities from the first infrared renormalon are canceled by ambiguities in the matrix elements that contribute through relative order $v^{2}$. We also deduce the leading renormalons for the decay $J / \psi \rightarrow \gamma \gamma \gamma$. Finally, in section V, we use our calculations to estimate higher order radiative corrections to the decay rates for $J / \psi \rightarrow e^{+} e^{-}, \eta_{c} \rightarrow \gamma \gamma$, and $J / \psi \rightarrow \gamma \gamma \gamma$.

\section{SHORT DISTANCE COEFFICIENTS AT TREE LEVEL}

In this section, we review the NRQCD factorization formalism as it applies to electromagnetic annihilation decays. We then calculate tree-level short-distance coefficients for the decays $J / \psi \rightarrow e^{+} e^{-}$and $\eta_{c} \rightarrow \gamma \gamma$ and for several quarkonium-to-vacuum matrix elements.

\section{A. NRQCD Factorization Formalism}

In the NRQCD factorization formalism, the annihilation decay rate for the charmonium state $H$ is written in the factorized form [1]

$$
\Gamma(H)=\frac{1}{2 M_{H}} \sum_{m n} C_{m n}\left\langle H\left|\mathcal{O}_{m n}\right| H\right\rangle,
$$

where $M_{H}$ is the mass of the state $H$. The matrix elements $\left\langle H\left|\mathcal{O}_{m n}\right| H\right\rangle$ are expectation values in the quarkonium state $H$ of local 4-fermion operators that have the structure

$$
\mathcal{O}_{m n}=\psi^{\dagger} \mathcal{K}_{m} \chi \chi^{\dagger} \mathcal{K}_{n} \psi
$$

where $\psi$ and $\chi$ are the field operators for the heavy quark and antiquark in NRQCD, $\mathcal{K}_{n}$ and $\mathcal{K}_{m}$ are products of a color matrix $\left(1\right.$ or $\left.T^{a}\right)$, a spin matrix $\left(1\right.$ or $\left.\sigma^{i}\right)$, and a polynomial in the gauge-covariant derivative $\mathbf{D}$ and in the field strengths $\mathbf{E}$ and $\mathbf{B}$. It is convenient to define the states $|H\rangle=|H(\mathbf{P}=0)\rangle$ in (11) so that they have the standard relativistic normalization:

$$
\left\langle H\left(\mathbf{P}^{\prime}\right) \mid H(\mathbf{P})\right\rangle=2 E_{P}(2 \pi)^{3} \delta^{3}\left(\mathbf{P}-\mathbf{P}^{\prime}\right),
$$

where $E_{P}=\sqrt{M_{H}^{2}+\mathbf{P}^{2}}$. The coefficients $C_{m n}$ take into account the effects of short distances of order $1 / m$, and they can therefore be calculated as perturbation series in the QCD coupling constant $\alpha_{s}(m)$.

For electromagnetic annihilation decays, the factorization formula can be simplified. Because there are no hadrons in the final state, one can insert a projection $|0\rangle\langle 0|$ onto the vacuum state between $\chi$ and $\chi^{\dagger}$ in the operator (2). The factorization formula (11) then becomes

$$
\Gamma(H \rightarrow \mathrm{EM})=\frac{1}{2 M_{H}} \sum_{m n} C_{m n}\left\langle H\left|\psi^{\dagger} \mathcal{K}_{m} \chi\right| 0\right\rangle\left\langle 0\left|\chi^{\dagger} \mathcal{K}_{n} \psi\right| H\right\rangle,
$$

where EM represents an electromagnetic final state consisting of photons or $e^{+} e^{-}$pairs. The matrix elements vanish unless the operators are color singlets, so $\mathcal{K}_{n}$ and $\mathcal{K}_{m}$ are products 
of a spin matrix $\left(1\right.$ or $\sigma^{i}$ ) and a polynomial in $\mathbf{D}, \mathbf{E}$, and $\mathbf{B}$. The short-distance coefficients $C_{m n}$ in (4) include the effects of integrating over the phase space of the electrons, positrons and photons in the final state. The NRQCD factorization can actually be carried out before the phase space integration at the level of the $T$-matrix element:

$$
\mathcal{T}_{H \rightarrow \mathrm{EM}}=\sum_{m} C_{m}\left\langle 0\left|\chi^{\dagger} \mathcal{K}_{m} \psi\right| H\right\rangle .
$$

The velocity-scaling rules of NRQCD can be used to determine how the matrix elements in (国) scale with the typical relative velocity $v$ of the heavy quark. For decays of the S-wave states $J / \psi$ and $\eta_{c}$, the leading contributions are from NRQCD matrix elements $\left\langle 0\left|\chi^{\dagger} \boldsymbol{\sigma} \psi\right| J / \psi\right\rangle$ and $\left\langle 0\left|\chi^{\dagger} \psi\right| \eta_{c}\right\rangle$, which scale like $v^{3 / 2}$. The next most important matrix elements are $\left\langle 0\left|\chi^{\dagger} \mathbf{D}^{2} \boldsymbol{\sigma} \psi\right| J / \psi\right\rangle$ and $\left\langle 0\left|\chi^{\dagger} \mathbf{D}^{2} \psi\right| \eta_{c}\right\rangle$, which are suppressed by $v^{2}$ and therefore represent relativistic corrections. The matrix elements are related by the approximate heavyquark spin symmetry of NRQCD:

$$
\begin{aligned}
\left\langle 0\left|\chi^{\dagger} \boldsymbol{\sigma} \psi\right| J / \psi(\boldsymbol{\epsilon})\right\rangle & =\boldsymbol{\epsilon}\left\langle 0\left|\chi^{\dagger} \psi\right| \eta_{c}\right\rangle\left(1+O\left(v^{2}\right)\right), \\
\left\langle 0\left|\chi^{\dagger} \mathbf{D}^{2} \boldsymbol{\sigma} \psi\right| J / \psi(\boldsymbol{\epsilon})\right\rangle & =\boldsymbol{\epsilon}\left\langle 0\left|\chi^{\dagger} \mathbf{D}^{2} \psi\right| \eta_{c}\right\rangle\left(1+O\left(v^{2}\right)\right),
\end{aligned}
$$

where $\boldsymbol{\epsilon}$ is the polarization vector of the $J / \psi$ which satisfies $\boldsymbol{\epsilon}^{2}=1$. Thus there are only 3 independent matrix elements that contribute to $S$-wave decays through relative order $v^{2}$.

The short-distance coefficients in the factorization formula (5) can be determined by the covariant projection method [12] or by the threshold expansion method [13]. In the threshold expansion method, the coefficients are determined by matching perturbative $T$ matrix elements for $c \bar{c}$ annihilation. Let $|c \bar{c}\rangle \equiv|c(\mathbf{q}, \xi) \bar{c}(-\mathbf{q}, \eta)\rangle$ represent a state that consists of a $c$ and a $\bar{c}$ with spatial momenta $\pm \mathbf{q}$ in the charmonium rest frame and with spin and color states specified by spinors $\xi$ and $\eta$ that are normalized to $\xi^{\dagger} \xi=\eta^{\dagger} \eta=1$. The standard relativistic normalization is

$$
\left\langle c\left(\mathbf{q}_{1}^{\prime}, \xi^{\prime}\right) \bar{c}\left(\mathbf{q}_{2}^{\prime}, \eta^{\prime}\right) \mid c\left(\mathbf{q}_{1}, \xi\right) \bar{c}\left(\mathbf{q}_{2}, \eta\right)\right\rangle=4 E_{q_{1}} E_{q_{2}}(2 \pi)^{6} \delta^{3}\left(\mathbf{q}_{1}-\mathbf{q}_{1}^{\prime}\right) \delta^{3}\left(\mathbf{q}_{2}-\mathbf{q}_{2}^{\prime}\right) \xi^{\prime \dagger} \xi \eta^{\prime \dagger} \eta,
$$

where $E_{q}=\sqrt{m^{2}+\mathbf{q}^{2}}$. In the threshold expansion method, the matching condition is

$$
\left.\mathcal{T}_{c \bar{c} \rightarrow \mathrm{EM}}\right|_{p Q C D}=\left.\sum_{m} C_{m}\left\langle 0\left|\chi^{\dagger} \mathcal{K}_{m} \psi\right| c \bar{c}\right\rangle\right|_{p N R Q C D},
$$

where $\mathcal{T}_{c \bar{c} \rightarrow \mathrm{EM}}$ is the $T$-matrix element for the annihilation of the $c \bar{c}$ into the electromagnetic final state EM. To carry out the matching procedure, the left side of (9) is calculated using perturbation theory in full QCD, and then expanded in powers of $\mathbf{q}$. The matrix elements on the right side of (9) are calculated using perturbation theory in NRQCD, and then expanded in powers of $\mathbf{q}$. The short-distance coefficients $C_{m}$ are obtained by matching the terms in the expansions in $\mathbf{q}$ order by order in $\alpha_{s}$. To determine all the coefficients $C_{m}$, it is also necessary to consider the annihilation of $c \bar{c}$ states with general momenta $\mathbf{q}_{1}$ and $\mathbf{q}_{2}$ and the annihilation of higher Fock states like $|c \bar{c} g\rangle$. At tree level, some of these coefficients can be determined more easily using an alternative method that involves a Foldy-Wouthuysen-Tani transformation. This method will be presented in the next subsections. 


\section{B. Leptonic Decay of $J / \psi$}

The decay $J / \psi \rightarrow e^{+} e^{-}$proceeds through the annihilation of the $J / \psi$ into a virtual photon. The Feynman diagram for $c \bar{c} \rightarrow e^{+} e^{-}$is shown in Fig. 1. The $T$-matrix element for the decay of $J / \psi$ into an electron and a positron with momenta $k_{1}$ and $k_{2}$ can be written as

$$
\mathcal{T}_{J / \psi \rightarrow e^{+} e^{-}}=-\frac{Q e^{2}}{M_{\psi}^{2}} \bar{u}\left(k_{1}\right) \gamma_{\mu} v\left(k_{2}\right)\left\langle 0\left|\bar{\Psi} \gamma^{\mu} \Psi\right| J / \psi\right\rangle,
$$

where $Q=+2 / 3$ is the electric charge of the charm quark and $M_{\psi}$ is the mass of the $J / \psi$. The factor of $1 / M_{\psi}^{2}$ comes from the propagator of the virtual photon. The matrix element in (10) is that of a local QCD operator between the $J / \psi$ and the vacuum state. Here and below, the argument of a local operator is assumed to be $x=0$ if it is not given explicitly. We obtain the decay width by squaring the $T$-matrix element, summing over the spins of the $e^{+}$and $e^{-}$, and integrating over the phase space of the $e^{+} e^{-}$pair:

$$
\Gamma\left(J / \psi \rightarrow e^{+} e^{-}\right)=\frac{4 \pi Q^{2} \alpha^{2}}{3 M_{\psi}^{3}}\left(-g_{\mu \nu}\right)\left\langle J / \psi\left|\bar{\Psi} \gamma^{\nu} \Psi\right| 0\right\rangle\left\langle 0\left|\bar{\Psi} \gamma^{\mu} \Psi\right| J / \psi\right\rangle .
$$

The QCD matrix element $\left\langle 0\left|\bar{\Psi} \gamma^{\mu} \Psi\right| J / \psi\right\rangle$ in (11) includes effects of both short distances of order $1 / m$ or smaller and long distances of order $1 / m v$ or larger. We can separate these effects by using the NRQCD factorization formalism to express the QCD matrix element as a sum of NRQCD matrix elements multiplied by short-distance coefficients. At tree level, this is most easily accomplished by a Foldy-Wouthuysen-Tani (FWT) transformation [14. This transformation can be constructed order by order in $v$ so that off-diagonal terms in the Hamiltonian that couple the heavy quark and antiquark are suppressed to any desired order in $v$. Up to corrections of order $v^{3}$, the FWT transformation is

$$
\Psi(x)=\exp \left(\frac{i}{2 m} \boldsymbol{\gamma} \cdot \mathbf{D}\right)\left(\begin{array}{c}
\psi(x) \\
\chi(x)
\end{array}\right),
$$

where $\mathbf{D}=\boldsymbol{\nabla}-i g_{s} \mathbf{A}$ is the covariant derivative. Inserting (12) into the matrix element $\left\langle 0\left|\bar{\Psi} \gamma^{\mu} \Psi\right| J / \psi\right\rangle$, expanding to second order in $\mathbf{D} / m$, and keeping only the annihilation terms that involve $\chi^{\dagger}$ and $\psi$, we obtain

$$
\left\langle 0\left|\bar{\Psi} \gamma^{\mu} \Psi\right| J / \psi\right\rangle=-g^{\mu i}\left[\left\langle 0\left|\chi^{\dagger} \sigma^{i} \psi\right| J / \psi\right\rangle+\frac{1}{4 m^{2}}\left\langle 0\left|\chi^{\dagger}\left(D_{i} D_{j}+D_{j} D_{i}\right) \sigma^{j} \psi\right| J / \psi\right\rangle\right] .
$$

Up to this point, the only property of the $J / \psi$ that we have used is that it has the quantum numbers $J^{P C}=1^{--}$that allow it to annihilate into a virtual photon. According to the velocity-scaling rules of NRQCD, the first term on the right side of (13) scales like $v^{3 / 2}$. The last term in (13) is a symmetric tensor in the indices of the two covariant derivatives. The trace of this tensor contributes at relative order $v^{2}$. The traceless part receives a contribution from the $D$-wave component of the $|c \bar{c}\rangle$ Fock state of the $J / \psi$ and is suppressed by $v^{4}$. Keeping only the terms in (13) that contribute through relative order $v^{2}$, this matrix element reads

$$
\left\langle 0\left|\bar{\Psi} \gamma^{\mu} \Psi\right| J / \psi\right\rangle=-g^{\mu i}\left[\left\langle 0\left|\chi^{\dagger} \sigma^{i} \psi\right| J / \psi\right\rangle+\frac{1}{6 m^{2}}\left\langle 0\left|\chi^{\dagger} \mathbf{D}^{2} \sigma^{i} \psi\right| J / \psi\right\rangle\right] .
$$


Inserting (14) into (11), the decay width can be written as

$$
\Gamma\left(J / \psi \rightarrow e^{+} e^{-}\right)=\frac{4 \pi Q^{2} \alpha^{2}}{3 M_{\psi}^{3}}\left|\left\langle 0\left|\chi^{\dagger} \boldsymbol{\sigma} \psi\right| J / \psi\right\rangle+\frac{1}{6 m^{2}}\left\langle 0\left|\chi^{\dagger} \mathbf{D}^{2} \boldsymbol{\sigma} \psi\right| J / \psi\right\rangle\right|^{2} .
$$

This is the NRQCD factorization formula for the decay width, including the first relativistic corrections and with the short-distance coefficients calculated to tree level.

In Ref. [1], the factorization formula for the decay rate of $J / \psi \rightarrow e^{+} e^{-}$was derived by matching $c \bar{c}$ scattering amplitudes. The $T$-matrix element for $c \bar{c} \rightarrow c \bar{c}$ through an intermediate $e^{+} e^{-}$state was matched with the $T$-matrix element for $c \bar{c} \rightarrow c \bar{c}$ through operators of the form $\psi^{\dagger} K_{m} \chi|0\rangle\langle 0| \chi^{\dagger} K_{n} \psi$ in NRQCD. Using our relativistic normalization of states and keeping only terms through relative order $v^{2}$, the result is

$$
\Gamma\left(J / \psi \rightarrow e^{+} e^{-}\right)=\frac{\pi Q^{2} \alpha^{2}}{3 M_{\psi} m^{2}}\left|\left\langle 0\left|\chi^{\dagger} \boldsymbol{\sigma} \psi\right| J / \psi\right\rangle+\frac{2}{3 m^{2}}\left\langle 0\left|\chi^{\dagger} \mathbf{D}^{2} \boldsymbol{\sigma} \psi\right| J / \psi\right\rangle\right|^{2} .
$$

As pointed out by Maksymyk [15], the consistency of (15) and (16) through relative order $v^{2}$ requires the identity

$$
\left\langle 0\left|\chi^{\dagger} \mathbf{D}^{2} \boldsymbol{\sigma} \psi\right| J / \psi\right\rangle=-m\left(M_{\psi}-2 m\right)\left\langle 0\left|\chi^{\dagger} \boldsymbol{\sigma} \psi\right| J / \psi\right\rangle\left(1+O\left(v^{2}\right)\right) .
$$

This identity was recently derived by Gremm and Kapustin [16], and follows simply from the equations of motion of NRQCD. It allows factors of $m$ in a factorization formula to be traded for factors of $M_{\psi}$ :

$$
\frac{M_{\psi}}{2 m}=1-\frac{1}{2 m^{2}} \frac{\boldsymbol{\epsilon} \cdot\left\langle 0\left|\chi^{\dagger} \mathbf{D}^{2} \boldsymbol{\sigma} \psi\right| J / \psi(\boldsymbol{\epsilon})\right\rangle}{\boldsymbol{\epsilon} \cdot\left\langle 0\left|\chi^{\dagger} \boldsymbol{\sigma} \psi\right| J / \psi(\boldsymbol{\epsilon})\right\rangle}+O\left(v^{4}\right) .
$$

The identity (18) can also be used to relate our NRQCD matrix elements, which are defined using the standard relativistic normalizations for the quarkonium states, to the matrix elements of Ref. [1], which were defined using the standard nonrelativistic normalizations:

$$
\left\langle 0\left|\chi^{\dagger} \mathcal{K}_{n} \psi\right| H\right\rangle=\sqrt{2 M_{H}}\left\langle 0\left|\chi^{\dagger} \mathcal{K}_{n} \psi\right| H\right\rangle_{\mathrm{BBL}}
$$

Using (18) and (19), we obtain the relation

$$
\begin{aligned}
\left\langle 0\left|\chi^{\dagger} \boldsymbol{\sigma} \psi\right| J / \psi\right\rangle=\sqrt{4 m} & \left(\left\langle 0\left|\chi^{\dagger} \boldsymbol{\sigma} \psi\right| J / \psi\right\rangle_{\mathrm{BBL}}\right. \\
& \left.-\frac{1}{4 m^{2}}\left\langle 0\left|\chi^{\dagger} \mathbf{D}^{2} \boldsymbol{\sigma} \psi\right| J / \psi\right\rangle_{\mathrm{BBL}}\right)\left(1+O\left(v^{4}\right)\right) .
\end{aligned}
$$

Inserting (20) into our factorization formula (15) and using (18) to eliminate $M_{\psi}$ in favor of $m$, we recover the result given in Ref. [1] up to corrections that are higher order in $v$ :

$$
\Gamma\left(J / \psi \rightarrow e^{+} e^{-}\right)=\frac{2 \pi Q^{2} \alpha^{2}}{3 m^{2}}\left|\left\langle 0\left|\chi^{\dagger} \boldsymbol{\sigma} \psi\right| J / \psi\right\rangle_{\mathrm{BBL}}+\frac{2}{3 m^{2}}\left\langle 0\left|\chi^{\dagger} \mathbf{D}^{2} \boldsymbol{\sigma} \psi\right| J / \psi\right\rangle_{\mathrm{BBL}}\right|^{2} .
$$




\section{Decay of $\eta_{c}$ into Photons}

If a quarkonium state $H$ is even under charge conjugation, it can decay into two photons at second order in the electromagnetic interaction. The Feynman diagrams for $c \bar{c} \rightarrow \gamma \gamma$ are shown in Fig. 2. If the photons have momenta $k_{1}$ and $k_{2}$ and polarization vectors $\boldsymbol{\epsilon}_{1}$ and $\boldsymbol{\epsilon}_{2}$, the $T$-matrix element for the decay is

$$
\begin{aligned}
\mathcal{T}_{H \rightarrow \gamma \gamma}= & -16 Q^{2} e^{2} \epsilon_{1 \mu} \epsilon_{2 \nu} \int d^{4} z e^{i\left(k_{1}-k_{2}\right) \cdot z} \\
& \times\left\langle 0\left|\left[\bar{\Psi}(z) \gamma^{\mu} S(z,-z) \gamma^{\nu} \Psi(-z)+\bar{\Psi}(-z) \gamma^{\nu} S(-z, z) \gamma^{\mu} \Psi(z)\right]\right| H\right\rangle .
\end{aligned}
$$

The factor of 16 arises from taking the integration variable $z$ to be half the separation of the interaction points. This expression involves the full propagator $S(x, y)$ of the heavy quark, which satisfies the equation

$$
(i \not D-m)_{x} S(x, y)=\delta^{4}(x-y)
$$

where $D_{\mu}=\partial_{\mu}+i g_{s} A_{\mu}$.

The phase factor in (22) and the behavior of the quark propagator combine to give the integral over $z$ support only in the region where the components of $z^{\mu}$ are of order of $1 / \mathrm{m}$. Since this is much smaller than the size $1 / m v$ of the quarkonium, the operator in (22) can be expanded in powers of $z^{\mu}$. It is convenient to carry out this expansion using the radial gauge [17]:

$$
z^{\mu} A_{\mu}(z)=0
$$

By Taylor expanding this gauge condition around $z=0$, one can show that derivatives of $A_{\mu}$ at the origin can be expressed in terms of gauge-invariant operators. In particular, we have $A_{\mu}(0)=0$ and $\partial_{\mu} A_{\nu}(0)=F_{\mu \nu}(0) / 2$. The quark field in the radial gauge can be expanded as

$$
\Psi(z)=\Psi(0)+z^{\mu} D_{\mu} \Psi(0)+\frac{1}{2} z^{\mu} z^{\nu} D_{\mu} D_{\nu} \Psi(0)+\cdots .
$$

By solving (23), we find that the quark propagator can be expanded as

$$
\begin{aligned}
S(z,-z) & =S_{F}(2 z)+\int d^{4} x S_{F}(z-x) g_{s} \not A(x) S_{F}(x+z)+\cdots \\
& =\int_{p} e^{-2 i p \cdot z}\left[\frac{1}{\not p-m}-\frac{i}{2} g_{s} F_{\mu \nu}(0) \frac{1}{\left(p^{2}-m^{2}\right)^{2}} \gamma^{\mu}(\not p-m) \gamma^{\nu}+\cdots\right],
\end{aligned}
$$

where $S_{F}(x)=\int_{p} e^{-i p \cdot x} /(\not p-m)$ is the Feynman propagator for a free quark. We have introduced an abbreviated notation for the integral over a four-momentum:

$$
\int_{p} \equiv \int \frac{d^{4} p}{(2 \pi)^{4}} .
$$

Inserting (25) and (26) into (22), expanding up to operators of dimension 5, and integrating over $z$, we obtain 


$$
\begin{aligned}
\mathcal{T}_{H \rightarrow \gamma \gamma}=-Q^{2} e^{2} \epsilon_{1 \mu} \epsilon_{2 \nu}\langle & 0 \mid \bar{\Psi}\left\{\frac{1}{r^{2}-m^{2}}\left(r_{\lambda} \Gamma_{-}^{\mu \lambda \nu}+2 m g^{\mu \nu}\right)\right. \\
& +i \frac{\partial}{\partial r_{\alpha}} \frac{1}{r^{2}-m^{2}}\left(r_{\lambda} \Gamma_{+}^{\mu \lambda \nu}+m \Gamma_{-}^{\mu \nu}\right) D_{\alpha} \\
& -\frac{1}{2} \frac{\partial}{\partial r_{\alpha}} \frac{\partial}{\partial r_{\beta}} \frac{1}{r^{2}-m^{2}}\left(r_{\lambda} \Gamma_{-}^{\mu \lambda \nu}+2 m g^{\mu \nu}\right) D_{\alpha} D_{\beta} \\
& \left.-\frac{1}{2} \frac{1}{\left(r^{2}-m^{2}\right)^{2}}\left(r_{\lambda} \Gamma_{+}^{\mu \alpha \lambda \beta \nu}-m \Gamma_{-}^{\mu \alpha \beta \nu}\right) i g_{s} F_{\alpha \beta}\right\} \Psi|H\rangle .
\end{aligned}
$$

We can set $r=\left(k_{1}-k_{2}\right) / 2$ and $r^{2}=-M_{H}^{2} / 4$ after taking the partial derivatives. We have used the notation

$$
\Gamma_{ \pm}^{\mu_{1} \mu_{2} \cdots \mu_{N}}=\gamma^{\mu_{1}} \gamma^{\mu_{2}} \cdots \gamma^{\mu_{N}} \pm \gamma^{\mu_{N}} \cdots \gamma^{\mu_{2}} \gamma^{\mu_{1}}
$$

To further simplify the $T$-matrix element, it is convenient to choose the photon polarization vectors to be space-like in the rest frame of the $H$. In that frame, we have $r_{0}=0, \mathbf{r} \cdot \boldsymbol{\epsilon}_{1}=0$, and $\mathbf{r} \cdot \boldsymbol{\epsilon}_{2}=0$. The FWT transformation (12) can be used to reduce $\mathcal{T}$ to a sum of NRQCD matrix elements. Keeping all terms through second order in $\mathbf{D} / m$, we obtain

$$
\begin{aligned}
\mathcal{T}_{H \rightarrow \gamma \gamma}=-i \frac{8 Q^{2} e^{2}}{M_{H}^{2}+4 m^{2}} \epsilon_{1}^{i} \epsilon_{2}^{j}\langle 0| \chi^{\dagger}\left\{\epsilon^{i j k} r^{k}+D_{i} \sigma^{j}+D_{j} \sigma^{i}+\frac{8 \delta^{i j} r^{m} r^{n}}{M_{H}^{2}+4 m^{2}} D_{m} \sigma^{n}\right. \\
+\frac{\left(M_{H}^{2}+12 m^{2}\right) \epsilon^{i j k} r^{k}}{2 m^{2}\left(M_{H}^{2}+4 m^{2}\right)} \mathbf{D}^{2}-\frac{64 \epsilon^{i j k} r^{k} r^{m} r^{n}}{\left(M_{H}^{2}+4 m^{2}\right)^{2}} D_{m} D_{n} \\
+\frac{\left(M_{H}^{2}-4 m^{2}\right) \epsilon^{i j k} r^{k}}{2 m^{2}\left(M_{H}^{2}+4 m^{2}\right)} g_{s} \mathbf{B} \cdot \boldsymbol{\sigma} \\
\left.-\frac{4 m}{M_{H}^{2}+4 m^{2}} g_{s}\left(E^{i} \sigma^{j}+E^{j} \sigma^{i}-\delta^{i j} \mathbf{E} \cdot \boldsymbol{\sigma}\right)\right\} \psi|H\rangle .
\end{aligned}
$$

To determine the coefficients of the matrix elements involving $\mathbf{B}$ and $\mathbf{E}$ using the threshold expansion method, we would have to match scattering amplitudes for $c \bar{c} g \rightarrow \gamma \gamma$.

Up to this point, the only property of the quarkonium state $H$ that we have used is that its charge conjugation quantum number is $C=+1$, so that it can decay into two photons. Thus the formula (30) applies equally well to the $\eta_{c}$, the $\chi_{c 0}$, and the $\chi_{c 2}$. We now specialize to the $\eta_{c}$, which has quantum numbers $J^{P C}=0^{-+}$. The parity conservation of NRQCD implies that the matrix elements of operators linear in $\mathbf{D}$ or linear in $\mathbf{E}$ are zero. Using the rotational invariance of NRQCD, the remaining matrix elements in (30) can be reduced to scalars:

$$
\begin{aligned}
\mathcal{T}_{\eta_{c} \rightarrow \gamma \gamma}=-i & \frac{4 Q^{2} e^{2} M_{\eta_{c}}}{M_{\eta_{c}}^{2}+4 m^{2}} \boldsymbol{\epsilon}_{1} \times \boldsymbol{\epsilon}_{2} \cdot \hat{\mathbf{r}} \\
& \times\left\{\left\langle 0\left|\chi^{\dagger} \psi\right| \eta_{c}\right\rangle+\frac{3 M_{\eta_{c}}^{4}+16 m^{2} M_{\eta_{c}}^{2}+144 m^{4}}{6 m^{2}\left(M_{\eta_{c}}^{2}+4 m^{2}\right)^{2}}\left\langle 0\left|\chi^{\dagger} \mathbf{D}^{2} \psi\right| \eta_{c}\right\rangle\right. \\
& \left.+\frac{M_{\eta_{c}}^{2}-4 m^{2}}{2 m^{2}\left(M_{\eta_{c}}^{2}+4 m^{2}\right)}\left\langle 0\left|\chi^{\dagger} g_{s} \mathbf{B} \cdot \boldsymbol{\sigma} \psi\right| \eta_{c}\right\rangle\right\}
\end{aligned}
$$


According to the velocity-scaling rules, the matrix element $\left\langle 0\left|\chi^{\dagger} \psi\right| \eta_{c}\right\rangle$ scales like $v^{3 / 2}$. The matrix element $\left\langle 0\left|\chi^{\dagger} \mathbf{D}^{2} \psi\right| \eta_{c}\right\rangle$ represents a relativistic correction that is suppressed by a power of $v^{2}$. The matrix element $\left\langle 0\left|\chi^{\dagger} g_{s} \mathbf{B} \cdot \boldsymbol{\sigma} \psi\right| \eta_{c}\right\rangle$ is suppressed by $v^{7 / 2}$. This suppression factor can be deduced using the methods described in Ref. [2]. In Coulomb gauge, the leading contribution to this matrix element comes from a $|c \bar{c} g\rangle$ Fock state, with the $c \bar{c}$ in a color-octet ${ }^{3} S_{1}$ state. This Fock state is dominated by dynamical gluons with momenta of order $m v$ and has a probability of order $v^{3}$. Relative to $\left\langle 0\left|\chi^{\dagger} \psi\right| \eta_{c}\right\rangle$, the matrix element $\left\langle 0\left|\chi^{\dagger} \mathbf{B} \cdot \boldsymbol{\sigma} \psi\right| \eta_{c}\right\rangle$ scales like $(m v)^{2} v^{3 / 2}$, with the factor of $v^{3 / 2}$ coming from the amplitude for the $c \bar{c} g$ Fock state and the factor of $(m v)^{2}$ coming from the dimension of the operator $\mathbf{B}$. Thus the suppression factor is $v^{7 / 2}$. Keeping only the terms through relative order $v^{2}$, the $T$-matrix element reduces to

$$
\begin{aligned}
\mathcal{T}_{\eta_{c} \rightarrow \gamma \gamma}= & -i \frac{4 Q^{2} e^{2} M_{\eta_{c}}}{M_{\eta_{c}}^{2}+4 m^{2}} \boldsymbol{\epsilon}_{1} \times \boldsymbol{\epsilon}_{2} \cdot \hat{\mathbf{r}} \\
& \times\left\{\left\langle 0\left|\chi^{\dagger} \psi\right| \eta_{c}\right\rangle+\frac{3 M_{\eta_{c}}^{4}+16 m^{2} M_{\eta_{c}}^{2}+144 m^{4}}{6 m^{2}\left(M_{\eta_{c}}^{2}+4 m^{2}\right)^{2}}\left\langle 0\left|\chi^{\dagger} \mathbf{D}^{2} \psi\right| \eta_{c}\right\rangle\right\} .
\end{aligned}
$$

We obtain the decay rate by squaring the $T$-matrix element, summing over the polarizations of the photons, and integrating over their phase space:

$$
\begin{aligned}
\Gamma\left(\eta_{c} \rightarrow \gamma \gamma\right)= & \frac{16 \pi Q^{4} \alpha^{2} M_{\eta_{c}}}{\left(M_{\eta_{c}}^{2}+4 m^{2}\right)^{2}} \\
& \times\left|\left\langle 0\left|\chi^{\dagger} \psi\right| \eta_{c}\right\rangle+\frac{3 M_{\eta_{c}}^{4}+16 m^{2} M_{\eta_{c}}^{2}+144 m^{4}}{6 m^{2}\left(M_{\eta_{c}}^{2}+4 m^{2}\right)^{2}}\left\langle 0\left|\chi^{\dagger} \mathbf{D}^{2} \psi\right| \eta_{c}\right\rangle\right|^{2} .
\end{aligned}
$$

The factorization formula (33) can be greatly simplified by using the identity analogous to (18) for the $\eta_{c}$ :

$$
\frac{M_{\eta_{c}}}{2 m}=1-\frac{1}{2 m^{2}} \frac{\left\langle 0\left|\chi^{\dagger} \mathbf{D}^{2} \psi\right| \eta_{c}\right\rangle}{\left\langle 0\left|\chi^{\dagger} \psi\right| \eta_{c}\right\rangle}+O\left(v^{4}\right) .
$$

Eliminating the quark mass from the coefficient of the leading matrix element in (33) and keeping only terms through relative order $v^{2}$, we get

$$
\Gamma\left(\eta_{c} \rightarrow \gamma \gamma\right)=\frac{4 \pi Q^{4} \alpha^{2}}{M_{\eta_{c}}^{3}}\left|\left\langle 0\left|\chi^{\dagger} \psi\right| \eta_{c}\right\rangle+\frac{1}{6 m^{2}}\left\langle 0\left|\chi^{\dagger} \mathbf{D}^{2} \psi\right| \eta_{c}\right\rangle\right|^{2} .
$$

The matrix elements can be expressed in terms of those introduced in Ref. [1] by using (19). Using (34) to eliminate the remaining factors of $M_{\eta_{c}}$ in favor of the quark mass, we recover the factorization formula derived in Ref. [1] up to corrections that are higher order in $v$ :

$$
\Gamma\left(\eta_{c} \rightarrow \gamma \gamma\right)=\frac{2 \pi Q^{4} \alpha^{2}}{m^{2}}\left|\left\langle 0\left|\chi^{\dagger} \psi\right| \eta_{c}\right\rangle_{\mathrm{BBL}}+\frac{2}{3 m^{2}}\left\langle 0\left|\chi^{\dagger} \mathbf{D}^{2} \psi\right| \eta_{c}\right\rangle_{\mathrm{BBL}}\right|^{2}
$$




\section{Quarkonium-to-Vacuum Matrix Elements}

In order to deduce the renormalon ambiguities in the NRQCD matrix elements in the factorization formulas (15) and (35), it will prove useful to also consider quarkonium-tovacuum matrix elements for all the QCD operators with dimension 3. A complete set of these operators is the scalar density $\bar{\Psi} \Psi$, the vector current $\bar{\Psi} \gamma^{\mu} \Psi$, the tensor current $\bar{\Psi} \sigma^{\mu \nu} \Psi$, the axial vector current $\bar{\Psi} \gamma^{\mu} \gamma_{5} \Psi$, and the pseudoscalar density $\bar{\Psi} \gamma_{5} \Psi$. Using the FWT transformation (12), we expand the matrix elements of each of these operators in terms of NRQCD matrix elements. Keeping terms up to second order in $\mathbf{D}$, the matrix elements for a general quarkonium state $H$ are

$$
\begin{aligned}
\langle 0|\bar{\Psi} \Psi| H\rangle & =\frac{i}{m}\left\langle 0\left|\chi^{\dagger} \mathbf{D} \cdot \boldsymbol{\sigma} \psi\right| H\right\rangle \\
\left\langle 0\left|\bar{\Psi} \gamma^{\mu} \Psi\right| H\right\rangle & =\delta^{\mu i}\left\langle 0\left|\chi^{\dagger}\left[\sigma^{i}+\frac{1}{4 m^{2}}\left(D_{i} D_{j}+D_{j} D_{i}\right) \sigma^{j}\right] \psi\right| H\right\rangle, \\
\left\langle 0\left|\bar{\Psi} \gamma^{\mu} \gamma_{5} \Psi\right| H\right\rangle & =\delta^{\mu 0}\left\langle 0\left|\chi^{\dagger}\left[1+\frac{1}{2 m^{2}}\left(\mathbf{D}^{2}+g_{s} \mathbf{B} \cdot \boldsymbol{\sigma}\right)\right] \psi\right| H\right\rangle \\
& +\delta^{\mu i} \frac{1}{m}\left\langle 0\left|\chi^{\dagger}(\mathbf{D} \times \boldsymbol{\sigma})^{i} \psi\right| H\right\rangle, \\
\left\langle 0\left|\bar{\Psi} \sigma^{\mu \nu} \Psi\right| H\right\rangle & =\delta^{\mu i} \delta^{\nu j} \frac{i}{m} \epsilon^{i j k}\left\langle 0\left|\chi^{\dagger} D_{k} \psi\right| H\right\rangle-i\left(\delta^{\mu 0} \delta^{\nu i}-\delta^{\nu 0} \delta^{\mu i}\right) \\
& \times\left\langle 0\left|\chi^{\dagger}\left[\sigma^{i}+\frac{1}{4 m^{2}}\left(2 \mathbf{D}^{2} \sigma^{i}-\left(D_{i} D_{j}+D_{j} D_{i}\right) \sigma^{j}+2 g_{s} B^{i}\right)\right] \psi\right| H\right\rangle \\
\left\langle 0\left|\bar{\Psi} \gamma^{5} \Psi\right| H\right\rangle & =-\left\langle 0\left|\chi^{\dagger} \psi\right| H\right\rangle .
\end{aligned}
$$

We now specialize to the $J / \psi$ and the $\eta_{c}$. For the $J / \psi$, the only operators that have matrix elements of order $v^{3 / 2}$ are the vector current, whose matrix element is given in (14), and the tensor current. Keeping only term through relative order $v^{2}$, the matrix element of the tensor current is

$$
\left\langle 0\left|\bar{\Psi} \sigma^{\mu \nu} \Psi\right| J / \psi\right\rangle=-i\left(\delta^{\mu 0} \delta^{\nu i}-\delta^{\nu 0} \delta^{\mu i}\right)\left[\left\langle 0\left|\chi^{\dagger} \sigma^{i} \psi\right| J / \psi\right\rangle+\frac{1}{3 m^{2}}\left\langle 0\left|\chi^{\dagger} \mathbf{D}^{2} \sigma^{i} \psi\right| J / \psi\right\rangle\right] .
$$

For the $\eta_{c}$, the operators that have matrix elements of order $v^{3 / 2}$ are the axial vector current and the pseudoscalar density. Keeping only term through relative order $v^{2}$, these matrix elements are

$$
\begin{aligned}
\left\langle 0\left|\bar{\Psi} \gamma^{\mu} \gamma_{5} \Psi\right| \eta_{c}\right\rangle & =\delta^{\mu 0}\left[\left\langle 0\left|\chi^{\dagger} \psi\right| \eta_{c}\right\rangle+\frac{1}{2 m^{2}}\left\langle 0\left|\chi^{\dagger} \mathbf{D}^{2} \psi\right| \eta_{c}\right\rangle\right] \\
\left\langle 0\left|\bar{\Psi} \gamma^{5} \Psi\right| \eta_{c}\right\rangle & =-\left\langle 0\left|\chi^{\dagger} \psi\right| \eta_{c}\right\rangle
\end{aligned}
$$

\section{SHORT-DISTANCE COEFFICIENTS IN THE LARGE- $N_{F}$ LIMIT}

In this section, we briefly review the Borel resummation method and the standard prescription for calculating the Borel transform of a perturbation series in QCD in the large- $N_{f}$ 
limit. We apply this prescription to the pole mass to illustrate the renormalization of the Borel transform. We then use the threshold expansion method to calculate the Borel transforms of the short-distance coefficients of the leading matrix elements in the NRQCD factorization formulas for $\left\langle 0\left|\bar{\Psi} \gamma^{\mu} \Psi\right| J / \psi\right\rangle,\left\langle 0\left|\bar{\Psi} \sigma^{\mu \nu} \Psi\right| J / \psi\right\rangle,\left\langle 0\left|\bar{\Psi} \gamma^{\mu} \gamma_{5} \Psi\right| \eta_{c}\right\rangle,\left\langle 0\left|\bar{\Psi} \gamma_{5} \Psi\right| \eta_{c}\right\rangle$, and the decay amplitude for $\eta_{c} \rightarrow \gamma \gamma$.

\section{A. Borel Resummation}

If a function $f\left(\alpha_{s}\right)$ has the power series expansion

$$
f\left(\alpha_{s}\right)=\sum_{n=0}^{\infty} a_{n} \alpha_{s}^{n},
$$

its Borel transform is defined by

$$
\widetilde{f}(t)=a_{0} \delta(t)+\sum_{n=1}^{\infty} \frac{1}{(n-1) !} a_{n} t^{n-1} .
$$

Obviously, the power series (46) for the Borel transform has better convergence properties than that of $f$. The original function $f$ can be recovered from its Borel transform $\widetilde{f}(t)$ by the inverse Borel transformation:

$$
f\left(\alpha_{s}\right)=\int_{0}^{\infty} d t e^{-t / \alpha_{s}} \tilde{f}(t)
$$

If the integral (47) is sufficiently convergent, it defines a unique function with the power series expansion (45) and the series is called Borel resummable.

A singularity in the Borel parameter $t$ is called a renormalon [3]. The contribution to $f\left(\alpha_{s}\right)$ from a pole of $\tilde{f}(t)$ at $t=t^{*}$ with residue $R^{*}$ can be obtained by replacing $\tilde{f}(t)$ in (47) by $R^{*} /\left(t-t^{*}\right)$. Expanding as a power series in $t$ and then integrating, we get

$$
f\left(\alpha_{s}\right) \sim-R^{*} \sum_{n}(n-1) !\left(\frac{\alpha_{s}}{t^{*}}\right)^{n} .
$$

Thus a renormalon gives rise to $n$ ! growth of the coefficients in the power series for $f\left(\alpha_{s}\right)$.

If the renormalon is on the negative real axis, this contribution to the series is Borel resummable. If the renormalon is on the positive real axis, there is an ambiguity in the function $f\left(\alpha_{s}\right)$. The possible prescriptions for the inverse Borel transform include deforming the integration contour in (47) into the complex plane so that it runs above the pole or below the pole. We also can take a linear combination of these two contours, such as the principle value. These prescriptions all differ by amounts that are proportional to the residue of the integrand of (47) at the pole. If the pole in $\widetilde{f}(t)$ is at the point $t^{*}$ and has residue $R^{*}$, the ambiguity in $f\left(\alpha_{s}\right)$ has the form

$$
\Delta f\left(\alpha_{s}\right)=K\left(2 \pi R^{*}\right) e^{-t^{*} / \alpha_{s}},
$$

where $K$ is an arbitrary constant. If $\alpha_{s}$ is the running coupling constant of QCD, its scale dependence is approximately 


$$
\alpha_{s}(Q) \approx \frac{1}{\beta_{0} \ln \left(Q^{2} / \Lambda_{\mathrm{QCD}}^{2}\right)},
$$

where $\beta_{0}=\left(33-2 N_{f}\right) / 12 \pi$. Inserting this into (49), we see that the renormalon ambiguity can be approximated by

$$
\Delta f \approx K\left(2 \pi R^{*}\right)\left(\frac{\Lambda_{\mathrm{QCD}}^{2}}{Q^{2}}\right)^{\beta_{0} t^{*}}
$$

Thus the ambiguity is suppressed by a power of $Q^{2}$.

To study the renormalons in NRQCD factorization formulas, we need to calculate the short-distance coefficients to all orders in $\alpha_{s}$. An exact calculation of the coefficients to all orders would be impossible. However the calculation is tractable in the large- $N_{f}$ limit of QCD, where $N_{f}$ is the number of light quarks [5]. The diagrams that dominate in this limit are obtained by inserting a chain of 1-loop quark bubbles into the gluon propagator in the lowest order diagrams that contain a gluon. All other diagrams are suppressed by a factor of $1 / N_{f}$. QCD is no longer an asymptotically free theory when $N_{f} \rightarrow \infty$, so it is more appropriate to consider the limit $N_{f} \rightarrow-\infty$. To be explicit, we define the large- $N_{f}$ limit to be the limit $N_{f} \rightarrow-\infty, \alpha_{s} \rightarrow 0$, with $N_{f} \alpha_{s}$ fixed.

The insertion of a light quark bubble renormalized by minimal subtraction into the propagator of a gluon of momentum $l$ gives a multiplicative factor

$$
-\beta_{0} \alpha_{s}\left[\frac{g(\epsilon)}{\epsilon}\left(\frac{-l^{2}-i \epsilon}{\mu^{2} e^{-\Delta}}\right)^{-\epsilon}-\frac{1}{\epsilon}\right] \frac{-l^{2} g^{\mu \nu}+l^{\mu} l^{\nu}}{l^{2}+i \epsilon},
$$

where $\beta_{0}=-N_{f} / 6 \pi$ is the first coefficient of the QCD beta function in the large- $N_{f}$ limit,

$$
g(\epsilon)=\frac{6(4 \pi)^{\epsilon} \Gamma(1+\epsilon) \Gamma^{2}(2-\epsilon)}{\Gamma(4-2 \epsilon)},
$$

and

$$
\Delta=-\gamma+\ln (4 \pi)+\frac{5}{3}+C
$$

The constant $C$ depends on the renormalization scheme and has the value $C=-5 / 3$ in the $\overline{\mathrm{MS}}$ scheme. A chain of bubble diagrams forms a geometric series. The Borel transform for this series multiplied by $\alpha_{s}$ can be obtained by making the following substitution for the gluon propagator in covariant gauge:

$$
\begin{aligned}
& \alpha_{s}\left[\frac{-g^{\mu \nu}}{l^{2}+i \epsilon}+(1-\xi) \frac{l^{\mu} l^{\nu}}{\left(l^{2}+i \epsilon\right)^{2}}\right] \\
& \longrightarrow \exp \left\{u\left[\frac{g(\epsilon)}{\epsilon}\left(\frac{-l^{2}-i \epsilon}{\mu^{2} e^{-\Delta}}\right)^{-\epsilon}-\frac{1}{\epsilon}\right]\right\} \frac{-l^{2} g^{\mu \nu}+l^{\mu} l^{\nu}}{\left(-l^{2}-i \epsilon\right)^{2}}-\xi \frac{l^{\mu} l^{\nu}}{\left(-l^{2}-i \epsilon\right)^{2}} \\
& =\left.\exp \left\{u\left[\frac{g(\epsilon)}{\epsilon} e^{\epsilon\left(\partial / \partial u^{\prime}\right)}-\frac{\partial}{\partial u^{\prime}}-\frac{1}{\epsilon}\right]\right\}\left(\frac{\mu^{2}}{e^{\Delta}}\right)^{u^{\prime}} \frac{g^{\mu \nu}}{\left(-l^{2}-i \epsilon\right)^{1+u^{\prime}}}\right|_{u^{\prime}=u}+l^{\mu} l^{\nu} \text { terms }
\end{aligned}
$$


where $u=\beta_{0} t$ and $t$ is the Borel parameter. The diagrams for electromagnetic decays considered in this paper are QED diagrams up to color factors. The contributions from the $l^{\mu} l^{\nu}$ terms in (55) cancel by the Ward identities of QED. The calculations can therefore be greatly simplified by using a propagator whose Lorentz structure is that of the gluon propagator in the Feynman gauge. The substitution (55) then reduces to

$$
\alpha_{s} \frac{-g^{\mu \nu}}{l^{2}+i \epsilon} \longrightarrow \mathcal{J}_{\epsilon}\left(\frac{\mu^{2}}{e^{C}}\right)^{u} \frac{g^{\mu \nu}}{\left(-l^{2}-i \epsilon\right)^{1+u}},
$$

where the action of the operator $\mathcal{J}_{\epsilon}$ on a function $f(u)$ is defined by

$$
\left.\mathcal{J}_{\epsilon} f(u) \equiv \exp \left\{u\left[\frac{g(\epsilon)}{\epsilon} e^{\epsilon\left(\partial / \partial u^{\prime}\right)}-\frac{\partial}{\partial u^{\prime}}-\frac{1}{\epsilon}\right]\right\} e^{-(\Delta-C) u^{\prime}} f\left(u^{\prime}\right)\right|_{u^{\prime}=u} .
$$

This operator is defined so that it reduces to the identity operator in the limit $\epsilon \rightarrow 0$ if it acts on a function with no poles in $\epsilon$.

In calculating the Borel transform of a sum of bubble chain diagrams, the extra factor $1 /\left(-l^{2}\right)^{u}$ introduced in the gluon propagator can introduce divergences in the loop integral that do not appear in the one-loop diagram, which corresponds to $u=0$. Below certain critical values of $u$, new power ultraviolet divergences appear. At such a value $u^{*}$, the loop integral has a logarithmic divergence, which appears as a pole in the Borel transform at $u=u^{*}$. This pole is an ultraviolet renormalon. Similarly, there are power infrared divergences that appear for $u$ above certain critical values $u^{*}$. At $u=u^{*}$, the loop integral is logarithmically infrared divergent. This logarithmic divergence appears as a pole in the Borel transform at $u=u^{*}$, and this is an infrared renormalon.

\section{B. Pole mass and renormalization constant}

We first calculate the Borel transform of the pole mass and the wave function renormalization constant of the heavy quark. The self-energy for a heavy quark with momentum $p$ can be expressed as a function of the coupling constant $\alpha_{s}$, the matrix $\not p$, and the bare mass $m_{0}$. We denote the self-energy by $\Sigma\left(\not p, \alpha_{s}\right)$, suppressing the dependence on $m_{0}$. In perturbation theory, the pole mass $m_{\text {pole }}\left(\alpha_{s}\right)$ is defined by the pole in the heavy quark propagator:

$$
m_{\text {pole }}\left(\alpha_{s}\right)=m_{0}+\left.\Sigma\left(\not p, \alpha_{s}\right)\right|_{\not p=m_{\text {pole }}\left(\alpha_{s}\right)} .
$$

One can solve the equation for $m_{\text {pole }}$ order by order in $\alpha_{s}$. The on-shell wavefunction renormalization constant $Z\left(\alpha_{s}\right)$ is given by $Z^{-1}=1-\delta Z$, where

$$
\delta Z\left(\alpha_{s}\right)=\left.\frac{\partial \Sigma\left(\not p, \alpha_{s}\right)}{\partial \not p}\right|_{\not p=m_{\text {pole }}\left(\alpha_{s}\right)} .
$$

A one-loop Feynman diagram for the heavy-quark self-energy with a chain of light quark bubbles inserted is shown in Fig. 3. Using the substitution (56), the Borel transform of the sum of the diagrams in Fig. 3 is given by 


$$
\widetilde{\Sigma}(\not p, t)=i \frac{16 \pi}{3} \mathcal{J}_{\epsilon}\left(\frac{\mu^{2}}{e^{C}}\right)^{u} \int_{l} \frac{1}{\left(-l^{2}-i \epsilon\right)^{1+u}} \gamma^{\mu} \frac{1}{\not p+\not l-m_{0}+i \epsilon} \gamma_{\mu}
$$

where the operator $\mathcal{J}_{\epsilon}$ is defined in (57). To regularize the ultraviolet divergence, we use dimensional regularization in $D=4-2 \epsilon$ dimensions. The integration measure in (60) is

$$
\int_{l} \equiv\left(\frac{\mu^{2}}{e^{\Delta}}\right)^{\epsilon} \int \frac{d^{D} l}{(2 \pi)^{D}}
$$

The $\epsilon$-dependent factor in front of the integral has been inserted so that renormalization by minimal subtraction will give the same renormalization scheme parametrized by the constant $C$ used in (52). The integral in (60) can be reduced to an integral over a Feynman parameter. For the Borel transforms of the pole mass in (58) and the wavefunction renormalization constant in (59), the integral can be expressed in terms of gamma functions:

$$
\begin{aligned}
\tilde{m}_{\text {pole }}(t) & =m_{0}\left[\delta(t)+\frac{3-2 \epsilon}{3 \pi} e^{(\gamma-5 / 3) \epsilon} \mathcal{J}_{\epsilon}\left(\frac{\mu^{2}}{e^{C} m_{0}^{2}}\right)^{u+\epsilon} \frac{\Gamma(u+\epsilon) \Gamma(3-2 u-2 \epsilon)}{(1-2 u-2 \epsilon) \Gamma(3-u-2 \epsilon)}\right] \\
\delta \widetilde{Z}(t) & =-\frac{3-2 \epsilon}{3 \pi} e^{(\gamma-5 / 3) \epsilon} \mathcal{J}_{\epsilon}\left(\frac{\mu^{2}}{e^{C} m_{0}^{2}}\right)^{u+\epsilon} \frac{(1+u) \Gamma(u+\epsilon) \Gamma(3-2 u-2 \epsilon)}{(1-2 u-2 \epsilon) \Gamma(3-u-2 \epsilon)}
\end{aligned}
$$

The expressions (62) and (63) are ultraviolet divergent and require renormalization. A renormalization procedure for the large- $N_{f}$ limit has been derived by Palanques-Mestre and Pascual [18] and by Broadhurst [19], and applied to the renormalization of the Borel transform by Beneke and Braun [8]. We can simplify their discussion since the renormalization of the light quark bubble diagram has already been carried out in (52). The ultraviolet divergences in (62) and (63) appear as poles in $\epsilon$ in the coefficients obtained by expanding these expressions as power series in $u$. The poles in $\epsilon$ arise from the power series expansion of the factor $\Gamma(u+\epsilon)$. In the minimal subtraction renormalization scheme, renormalization is accomplished by subtracting the pole terms from the coefficients and then taking the limit $\epsilon \rightarrow 0$. The functions of $u$ in (62) and (63) can be expressed in the form

$$
\widetilde{f}(u, \epsilon)=\mathcal{J}_{\epsilon} \frac{F(u+\epsilon, \epsilon)}{u+\epsilon},
$$

where $F(u+\epsilon, \epsilon)$ is analytic in $u+\epsilon$ at $u+\epsilon=0$. The renormalization of this expression by minimal subtraction involves subtracting the poles in $\epsilon$ from the coefficients in its power series expansion in $u$, followed by taking the limit $\epsilon \rightarrow 0$. The final result is rather simple:

$$
\widetilde{f}_{M S}(u)=\frac{1}{u}[F(u, 0)-\widetilde{G}(u)]
$$

where $\widetilde{G}(u)$ is the Borel transform of the function

$$
G(x)=\frac{(4 \pi)^{x} \Gamma(4+2 x)}{6 \Gamma(1-x) \Gamma^{2}(2+x)} F(0,-x) .
$$

We can derive this result through several simple steps that allow us to take the limit $\epsilon \rightarrow 0$ in parts of the expression (64). The first step is to subtract $e^{(\Delta-C) \epsilon} F(0, \epsilon) /\left(u^{\prime}+\epsilon\right)$ from 
$e^{-(\Delta-C) u^{\prime}} F\left(u^{\prime}+\epsilon, \epsilon\right) /\left(u^{\prime}+\epsilon\right)$ in (64), and add it back in again. The subtraction cancels the pole at $u^{\prime}+\epsilon=0$, so we can take the limit $\epsilon \rightarrow 0$ except in the added term:

$$
\begin{aligned}
\tilde{f}(u, \epsilon) \longrightarrow & \frac{F(u, 0)-e^{u(\Delta-C)} F(0,0)}{u} \\
& +\left.F(0, \epsilon) e^{-u / \epsilon+(\Delta-C) \epsilon} \exp \left\{u\left[\frac{g(\epsilon)}{\epsilon} e^{\epsilon\left(\partial / \partial u^{\prime}\right)}-\frac{\partial}{\partial u^{\prime}}\right]\right\} \frac{1}{u^{\prime}+\epsilon}\right|_{u^{\prime}=u} .
\end{aligned}
$$

It is convenient to use the following integral representation for $1 /\left(u^{\prime}+\epsilon\right)$ :

$$
\frac{1}{u^{\prime}+\epsilon}=\int_{0}^{\infty} d \alpha e^{-\left(u^{\prime}+\epsilon\right) \alpha}
$$

Inside the integral, the action of the differential operator $\partial / \partial u^{\prime}$ is simply multiplication by $-\alpha$. After setting $u^{\prime}=u$, the action of the exponentiated operator in (67) can be carried out analytically:

$$
\left.\exp \left[u \frac{g(\epsilon)}{\epsilon} e^{\epsilon\left(\partial / \partial u^{\prime}\right)}-u \frac{\partial}{\partial u^{\prime}}\right] \frac{1}{u^{\prime}+\epsilon}\right|_{u^{\prime}=u}=\frac{1}{u g(\epsilon)}\left[e^{u g(\epsilon) / \epsilon}-1\right] .
$$

Inserting (69) into (67), we can take the limit $\epsilon \rightarrow 0$ in the term with the factor $e^{u g(\epsilon) / \epsilon}$. The resulting expression for the Borel transform is

$$
\tilde{f}(u, \epsilon) \longrightarrow \frac{1}{u}\left[F(u, 0)-\frac{F(0, \epsilon)}{g(\epsilon)} e^{-u / \epsilon+(\Delta-C) \epsilon}\right]
$$

Minimal subtraction can now be carried out by using the identity

$$
\left[G(-\epsilon) e^{-u / \epsilon}\right]_{M S}=\widetilde{G}(u),
$$

where $\widetilde{G}(u)$ is the Borel transform of the function $G(x)$. Inserting this into (70), we obtain our final result (65).

We now apply (65) to the expression (62) to obtain an expression for the Borel transform of the pole mass in terms of the running mass $m(\mu)$ defined by minimal subtraction:

$$
\tilde{m}_{\text {pole }}(t)=m(\mu)\left\{\delta(t)+\frac{1}{\pi u}\left[\left(\frac{\mu^{2}}{e^{C} m^{2}(\mu)}\right)^{u} \frac{\Gamma(1+u) \Gamma(3-2 u)}{(1-2 u) \Gamma(3-u)}-\widetilde{G}_{m}(u)\right]\right\},
$$

where $\widetilde{G}_{m}(u)$ is the Borel transform of the function

$$
G_{m}(x)=\frac{(3+2 x) \Gamma(4+2 x)}{9 \Gamma(1-x) \Gamma^{2}(2+x) \Gamma(3+x)} .
$$

This agrees with Eqs. (4.3) and (4.4) of Ref. [7]. The expression (72) for $\tilde{m}_{\text {pole }}$ has poles in the Borel parameter $t=u / \beta_{0}$. These poles are called renormalons. They are classified as ultraviolet renormalons or infrared renormalons according to whether they arise from the integration region of high loop momentum or low loop momentum. The pole in (72) from high loop momentum are those that appear in the factor $\Gamma(1+u)$. Thus there are ultraviolet renormalons in the pole mass at the negative integers $u=-1,-2, \cdots$. There are infrared renormalons at the positive half-integers $u=\frac{1}{2}, \frac{3}{2}, \cdots$, and also at $u=2$. From (63), we see that $\delta Z$ has renormalons at the same positions as the pole mass except that there is no renormalon at $u=-1$. 


\section{Quarkonium-to-Vacuum Matrix Elements}

Now we calculate the Borel transforms of the short-distance coefficients in the NRQCD factorization formulas for the quarkonium-to-vacuum matrix elements. We define the shortdistance coefficients $C^{\Gamma}\left(\alpha_{s}\right)$, where $\Gamma=V, A, T, P$, by the factorization formulas

$$
\begin{aligned}
\left\langle 0\left|\bar{\Psi} \gamma^{\mu} \Psi\right| J / \psi\right\rangle & =\delta^{\mu i}\left[1+C^{V}\left(\alpha_{s}\right)\right]\left\langle 0\left|\chi^{\dagger} \sigma^{i} \psi\right| J / \psi\right\rangle+\cdots, \\
\left\langle 0\left|\bar{\Psi} \gamma^{\mu} \gamma_{5} \Psi\right| \eta_{c}\right\rangle & =\delta^{\mu 0}\left[1+C^{A}\left(\alpha_{s}\right)\right]\left\langle 0\left|\chi^{\dagger} \psi\right| \eta_{c}\right\rangle+\cdots, \\
\left\langle 0\left|\bar{\Psi} \sigma^{\mu \nu} \Psi\right| J / \psi\right\rangle & =-i\left(\delta^{\mu 0} \delta^{\nu i}-\delta^{\nu 0} \delta^{\mu i}\right)\left[1+C^{T}\left(\alpha_{s}\right)\right]\left\langle 0\left|\chi^{\dagger} \sigma^{i} \psi\right| J / \psi\right\rangle+\cdots, \\
\left\langle 0\left|\bar{\Psi} \gamma_{5} \Psi\right| \eta_{c}\right\rangle & =-\left[1+C^{P}\left(\alpha_{s}\right)\right]\left\langle 0\left|\chi^{\dagger} \psi\right| \eta_{c}\right\rangle+\cdots .
\end{aligned}
$$

We use $\Gamma$ to represent the matrices $\gamma^{\mu}, \gamma^{\mu} \gamma_{5}, \sigma^{\mu \nu}$, and $\gamma_{5}$, so that the matrix elements on the left side of (74)-(77) are denoted genericly by $\langle 0|\bar{\Psi} \Gamma \Psi| H\rangle$.

The short-distance coefficients $C^{\Gamma}\left(\alpha_{s}\right)$ in (74)-(77) can be determined by matching the corresponding equations for $|c \bar{c}\rangle$ states computed in perturbation theory. The simplest choice is a state $|c \bar{c}\rangle$ that consist of a $c$ and $\bar{c}$ at rest. We must compute $\langle 0|\bar{\Psi} \Gamma \Psi| c \bar{c}\rangle$ using perturbative QCD and $\left\langle 0\left|\chi^{\dagger} \sigma^{i} \psi\right| c \bar{c}\right\rangle$ and $\left\langle 0\left|\chi^{\dagger} \psi\right| c \bar{c}\right\rangle$ using perturbative NRQCD, and then determine $C^{\Gamma}\left(\alpha_{s}\right)$ by matching the left and right sides of (74)-(77). By Borel transforming both sides of the equation before carrying out the matching, we can determine the Borel transform $\widetilde{C}^{\Gamma}(t)$.

We first consider the QCD side of the matching conditions. The $c$ and $\bar{c}$ at rest have equal four-momenta $p=(m, \mathbf{0})$ and are represented by the spinors

$$
u(p)=\sqrt{2 m}\left(\begin{array}{l}
\xi \\
0
\end{array}\right), \quad v(p)=\sqrt{2 m}\left(\begin{array}{l}
0 \\
\eta
\end{array}\right) .
$$

The diagrams that dominate the radiative corrections in the large- $N_{f}$ limit are obtained by inserting a chain of light quark bubbles into the gluon propagator in the one-loop diagrams. The diagrams with propagator corrections on the $c$ and $\bar{c}$ lines reduce to the tree level matrix element $\bar{v} \Gamma u$ multiplied by $\delta Z\left(\alpha_{s}\right)$. After Borel transforming, they give $\delta \widetilde{Z}(t) \bar{v} \Gamma u$. The remaining diagrams are vertex corrections, such as the diagram for $c \bar{c} \rightarrow e^{+} e^{-}$in Figure 4. The Borel transform of the vertex correction is

$$
i \frac{16 \pi}{3} \mathcal{J}_{\epsilon}\left(\frac{\mu^{2}}{e^{C}}\right)^{u} \int_{l} \frac{1}{\left(-l^{2}-i \epsilon\right)^{1+u}} \bar{v}(p) \gamma^{\nu} \frac{1}{\not-\not p-m+i \epsilon} \Gamma \frac{1}{\not+\not p-m+i \epsilon} \gamma_{\nu} u(p) .
$$

The dimensionally regularized integral in $(79)$ can be evaluated by introducing Feynman pa-

rameters. After a straightforward calculation, we find that (79) reduces to $\tilde{D}^{\Gamma}(t) \bar{v}(p) \Gamma u(p)$, where

$$
\begin{aligned}
\tilde{D}^{\Gamma}(t)= & \frac{2}{3 \pi} e^{(\gamma-5 / 3) \epsilon} \mathcal{J}_{\epsilon}\left(\frac{\mu^{2}}{e^{C} m^{2}}\right)^{u+\epsilon} \frac{\Gamma(u+\epsilon) \Gamma(1-2 u-2 \epsilon)}{(1+2 u+2 \epsilon) \Gamma(3-u-2 \epsilon)} \\
& \times \begin{cases}(1-2 \epsilon-u)[(1-\epsilon)(3+2 \epsilon)+(1-2 \epsilon) u] & \Gamma=V, \\
(3-2 \epsilon)\left[(1-\epsilon)+\epsilon u+u^{2}\right] & \Gamma=A, \\
2(1-\epsilon)(1-2 \epsilon)-\left(3-3 \epsilon+2 \epsilon^{2}\right) u+(1-2 \epsilon) u^{2} & \Gamma=T, \\
(3-2 \epsilon)(1+\epsilon+u)(2-2 \epsilon-u) & \Gamma=P,\end{cases}
\end{aligned}
$$


where the operator $\mathcal{J}_{\epsilon}$ acts on every function of $u$ to the right. The prescription we have used for $\gamma_{5}$ in $D$ dimensions is that $\left\{\gamma_{5}, \gamma^{\mu}\right\}=0$ for all $\mu$.

We next consider the NRQCD sides of the matching conditions. If we use dimensional regularization, the only scale in the perturbative NRQCD matrix elements is the relative momentum $\mathbf{q}$. Having set $\mathbf{q}=0$, the only matrix elements that survive are $\left\langle 0\left|\chi^{\dagger} \sigma^{i} \psi\right| c \bar{c}\right\rangle$ and $\left\langle 0\left|\chi^{\dagger} \psi\right| c \bar{c}\right\rangle$. The matrix elements of all higher dimension operators vanish. The radiative corrections to the matrix elements vanish in dimensional regularization, since there is no momentum scale in the loop integral. Thus the NRQCD matrix elements are trivial:

$$
\begin{aligned}
\left\langle 0\left|\chi^{\dagger} \sigma^{i} \psi\right| c \bar{c}\right\rangle & =2 m \eta^{\dagger} \sigma^{i} \xi, \\
\left\langle 0\left|\chi^{\dagger} \psi\right| c \bar{c}\right\rangle & =2 m \eta^{\dagger} \xi .
\end{aligned}
$$

Since these matrix elements are independent of $\alpha_{s}$, the Borel transforms of the right sides of (74)-(77) are obtained simply by replacing $1+C^{\Gamma}\left(\alpha_{s}\right)$ by $\delta(t)+\widetilde{C}^{\Gamma}(t)$.

By matching the Borel transforms of the QCD and NRQCD sides of (74)-(77), we find that the short-distance coefficients are given by

$$
\widetilde{C}^{\Gamma}(t)=\tilde{D}^{\Gamma}(t)+\delta \widetilde{Z}(t)
$$

where $\delta \widetilde{Z}(t)$ is given in (63) and $\tilde{D}^{\Gamma}(t)$ for $\Gamma=V, A, T$, and $P$ is given in (80). The Borel transforms $\widetilde{C}^{V}$ and $\widetilde{C}^{A}$ are finite at $u+\epsilon=0$ and therefore do not need to be renormalized. This is simply a consequence of the conservation of the vector current and the partial conservation of the axial current. We can therefore set $\epsilon=0$ in these coefficients:

$$
\begin{aligned}
& \widetilde{C}^{V}(t)=-\frac{2}{3 \pi}\left(\frac{\mu^{2}}{e^{C} m^{2}}\right)^{u} \frac{4+3 u}{(1-2 u)(1+2 u)} \frac{\Gamma(1+u) \Gamma(3-2 u)}{\Gamma(3-u)}, \\
& \widetilde{C}^{A}(t)=-\frac{4}{\pi}\left(\frac{\mu^{2}}{e^{C} m^{2}}\right)^{u} \frac{1-u-u^{2}}{1+2 u} \frac{\Gamma(1+u) \Gamma(1-2 u)}{\Gamma(3-u)} .
\end{aligned}
$$

In the Borel transforms $\widetilde{C}^{T}$ and $\widetilde{C}^{P}$, there is a pole at $u+\epsilon=0$. This pole indicates that there are ultraviolet divergences in the coefficients of the power series expansion in $u$. These divergences are removed by multiplicative renormalizations of the QCD operators $\bar{\Psi} \sigma^{\mu \nu} \Psi$ and $\bar{\Psi} \gamma_{5} \Psi$. If we use the minimal subtraction renormalization scheme, the renormalization can be carried out by making the substitution (65) [[ CHANGE EQ. NO. (65). ]] Our final results for these coefficients are

$$
\begin{aligned}
& \widetilde{C}^{T}(t)=-\frac{1}{3 \pi u}\left[\left(\frac{\mu^{2}}{e^{C} m^{2}}\right)^{u} \frac{1+10 u+6 u^{2}}{(1-2 u)(1+2 u)} \frac{\Gamma(1+u) \Gamma(3-2 u)}{\Gamma(3-u)}-\widetilde{G}^{T}(u)\right], \\
& \widetilde{C}^{P}(t)=\frac{2}{\pi u}\left[\left(\frac{\mu^{2}}{e^{C} m^{2}}\right)^{u} \frac{1-2 u+2 u^{2}}{1+2 u} \frac{\Gamma(2+u) \Gamma(1-2 u)}{\Gamma(3-u)}-\frac{1}{2} \widetilde{G}_{m}(u)\right],
\end{aligned}
$$

where $\widetilde{G}^{T}(u)$ is the Borel transform of the function

$$
G^{T}(x)=\frac{(1+2 x) \Gamma(4+2 x)}{3 \Gamma(1-x) \Gamma^{2}(2+x) \Gamma(3+x)}
$$

and $\widetilde{G}_{m}(u)$ is the Borel transform of the function $G_{m}(x)$ defined in (73). 
From (84)-(87), we see that all the coefficients have ultraviolet renormalons at the negative integers $u=-1,-2, \cdots$, except for $\widetilde{C}^{P}$, which does not have a renormalon at $u=-1$. They all have infrared renormalons at the positive half-integers $u=\frac{1}{2}, \frac{3}{2}, \cdots$, and at $u=2$. The coefficients $\widetilde{C}^{A}$ and $\widetilde{C}^{P}$ have an additional infrared renormalon at $u=1$. Finally, all the coefficients have a renormalon at $u=-\frac{1}{2}$. This appears to be an infrared renormalon in spite of the fact that it appears on the negative real axis, because it arises from the low momentum region of the integral over the loop momentum $l$ in (79). However the low momentum region of that integral is actually canceled by the low momentum region of an integral on the NRQCD side of the matching condition. The integral on the NRQCD side has the form

$$
-\frac{16 \pi m}{3}\left(\frac{\mu^{2}}{e^{\Delta}}\right)^{\epsilon} \int \frac{d^{3-2 \epsilon} l}{(2 \pi)^{3-2 \epsilon}} \frac{1}{\left(l^{2}\right)^{2+u}} .
$$

This vanishes in dimensional regularization due to a cancellation between an infrared pole at $u+\epsilon=-\frac{1}{2}$ and an ultraviolet pole at $u+\epsilon=-\frac{1}{2}$. Since the infrared pole is canceled by the QCD contribution to $\widetilde{C}^{\Gamma}$, the renormalon at $u=-\frac{1}{2}$ is actually an ultraviolet renormalon of NRQCD.

To obtain the power series expansions for the coefficients $C^{\Gamma}\left(\alpha_{s}\right)$, we expand the coefficients $\widetilde{C}^{\Gamma}(t)$ as a power series in $t$ and then invert the Borel transform by substituting $t^{n-1} \rightarrow(n-1) ! \alpha_{s}^{n}$. The first two terms in the expansion of $C^{V}\left(\alpha_{s}\right)$ are

$$
C^{V}\left(\alpha_{s}\right)=-\frac{8}{3} \frac{\alpha_{s}(\mu)}{\pi}+\left(2-\frac{16}{3} \log \frac{\mu}{e^{C / 2} m}\right) \frac{\beta_{0} \alpha_{s}^{2}}{\pi} .
$$

The coefficient of $\alpha_{s}$ was first calculated by Barbieri et al. [20]. From the $\alpha_{s}^{2}$ term in (90), we can read off the Brodsky-Lepage-Mackenzie (BLM) scale [21] for this process, which is the scale $\mu$ for which the correction proportional to $N_{f} \alpha_{s}^{2}$ vanishes. We find $\mu=1.46 e^{C / 2} m$.

\section{Decay of $\eta_{c}$ into $\gamma \gamma$}

We next calculate the Borel transform of the short-distance coefficient of $\left\langle 0\left|\chi^{\dagger} \psi\right| \eta_{c}\right\rangle$ in the NRQCD factorization formula for the $T$-matrix element for $\eta_{c} \rightarrow \gamma \gamma$. The factorization formula with tree-level coefficients is given in (32). Up to this point, it has not been necessary to define carefully the mass $m$ that appears in the factorization formula. It could equally well be the running mass $m(\mu)$ defined by a minimal subtraction renormalization scheme, or the pole mass $m_{\text {pole }}$ defined by the location of the pole in the propagator in perturbation theory, or any other mass that differs from these by perturbative corrections. However, in order to calculate the short-distance coefficient beyond tree level, it is necessary to specify the definition of $m$. The choice is somewhat arbitrary, since a different choice can be compensated by changes in the perturbative corrections to the short-distance coefficient. We choose to use the pole mass. We define the short-distance coefficient $C^{\gamma \gamma}\left(\alpha_{s}\right)$ by the factorization formula

$$
\mathcal{T}_{\eta_{c} \rightarrow \gamma \gamma}=-i \frac{4 Q^{2} e^{2} M_{\eta_{c}}}{M_{\eta_{c}}^{2}+4 m_{\text {pole }}^{2}} \boldsymbol{\epsilon}_{1} \times \boldsymbol{\epsilon}_{2} \cdot \hat{\mathbf{r}}\left[1+C^{\gamma \gamma}\left(\alpha_{s}\right)\right]\left\langle 0\left|\chi^{\dagger} \psi\right| \eta_{c}\right\rangle+\cdots
$$


Our choice of the pole mass is dictated primarily by the algebraic simplicity of the final result for the Borel transform of $C^{\gamma \gamma}\left(\alpha_{s}\right)$. The coefficient $C^{\gamma \gamma}$ in (91) can be determined by matching the perturbative $T$-matrix elements for $c \bar{c} \rightarrow \gamma \gamma$ in QCD and in NRQCD, as in (9). If we take the $c$ and $\bar{c}$ to be at rest, their invariant mass is $M_{c \bar{c}}=2 m_{\text {pole }}$, and the matching condition reduces to

$$
\left.\mathcal{T}_{c \bar{c} \rightarrow \gamma \gamma}\right|_{p Q C D}=-i \frac{Q^{2} e^{2}}{2 m_{\text {pole }}} \boldsymbol{\epsilon}_{1} \times\left.\boldsymbol{\epsilon}_{2} \cdot \hat{\mathbf{r}}\left[1+C^{\gamma \gamma}\left(\alpha_{s}\right)\right]\left\langle 0\left|\chi^{\dagger} \psi\right| c \bar{c}\right\rangle\right|_{p N R Q C D}+\cdots .
$$

By Borel transforming both sides of the equation, we can determine $\widetilde{C}^{\gamma \gamma}(t)$.

We first consider the NRQCD side of the matching condition. Having chosen the relative momentum of the $c \bar{c}$ pair to be 0 , the radiative corrections to the matrix element vanish in dimensional regularization. The matrix element therefore reduces to

$$
\left\langle 0\left|\chi^{\dagger} \psi\right| c \bar{c}\right\rangle=2 m_{\text {pole }} \eta^{\dagger} \xi
$$

The Borel transform of the right side of (92) is therefore obtained by replacing $1+C^{\gamma \gamma}\left(\alpha_{s}\right)$ by $\delta(t)+\widetilde{C}^{\gamma \gamma}(t)$.

We next consider the QCD side of the matching equation (92). Since the $c$ and $\bar{c}$ are at rest, they have equal 4-momentum $p=(m, \mathbf{0})$ and their spinors are given in (78). The diagrams for the $T$-matrix element in the large $N_{f}$ limit are those given in Figs. 5a-e, together with the diagrams with self-energy corrections on the external $c$ and $\bar{c}$ lines. In each of the diagrams, the spinor factor can be reduced to $r_{\lambda} \bar{v} \Gamma_{-}^{\mu \lambda \nu} u$, where $r=\left(k_{1}-k_{2}\right) / 2$. The contribution to $\widetilde{C}^{\gamma \gamma}(t)$ can be obtained from the Borel transform of the diagram by removing the factor

$$
\frac{Q^{2} e^{2}}{2 m^{2}} \epsilon_{1 \mu} \epsilon_{2 \nu} r_{\lambda} \bar{v}(p) \Gamma_{-}^{\mu \lambda \nu} u(p)=-i Q^{2} e^{2} \boldsymbol{\epsilon}_{1} \times \boldsymbol{\epsilon}_{2} \cdot \hat{\mathbf{r}} \eta^{\dagger} \xi
$$

The ultraviolet divergences associated with vertex corrections and wavefunction renormalizations cancel by the Ward identities of QED. The only renormalization that is necessary is therefore mass renormalization. It is convenient to use on-shell renormalization. Explicit regularization of the loop integrals is unnecessary, since the Borel parameter itself provides a regulator. The mass counterterm and the wavefunction renormalization constant can be obtained from (62) and (63) by setting $\epsilon=0$ :

$$
\begin{aligned}
& \delta \widetilde{m}(t)=m \frac{2}{\pi}\left(\frac{\mu^{2}}{e^{C} m^{2}}\right)^{u} \frac{1-u}{u} \frac{\Gamma(1-2 u) \Gamma(1+u)}{\Gamma(3-u)} \\
& \delta \widetilde{Z}(t)=-\frac{2}{\pi}\left(\frac{\mu^{2}}{e^{C} m^{2}}\right)^{u} \frac{(1+u)(1-u)}{u} \frac{\Gamma(1-2 u) \Gamma(1+u)}{\Gamma(3-u)} .
\end{aligned}
$$

The short-distance coefficient $\widetilde{C}^{\gamma \gamma}(t)$ can be written

$$
\widetilde{C}^{\gamma \gamma}(t)=\widetilde{C}_{1}(t)+\widetilde{C}_{2}(t)+\widetilde{C}_{3}(t)+\delta \widetilde{Z}(t)
$$

where $\widetilde{C}_{1}(t)$ is the contribution from the box diagrams in Fig. $5 \mathrm{a}, \widetilde{C}_{2}(t)$ is the contribution from the vertex corrections in Figs. $5 \mathrm{~b}$ and $5 \mathrm{c}, \widetilde{C}_{3}(t)$ is the sum of the contribution from 
the propagator correction in Fig. $5 \mathrm{~d}$ and the mass counterterm in Fig $5 \mathrm{e}$, and $\delta \widetilde{Z}(t)$ is the contribution from propagator corrections on the external lines.

The sum of the Borel transform of the box diagram in Fig. 5a and the diagram obtained by interchanging the photon lines is given by

$$
\begin{aligned}
& \frac{16 \pi Q^{2} e^{2}}{3} \epsilon_{1 \mu} \epsilon_{2 \nu}\left(\frac{\mu^{2}}{e^{C}}\right)^{u} \int_{l} \frac{1}{\left(-l^{2}\right)^{1+u}} \bar{v}(p) \gamma^{\alpha} \frac{1}{\not-\not p-m} \\
& \quad \times\left(\gamma^{\mu} \frac{1}{\not+\not p-m} \gamma^{\nu}+\gamma^{\nu} \frac{1}{\not-\not p-m} \gamma^{\mu}\right) \frac{1}{\not+\not p-m} \gamma_{\alpha} u(p) .
\end{aligned}
$$

The integral in Eq. (98) can be carried out using Feynman parameters. Removing the factor (94), we obtain

$$
\begin{aligned}
\widetilde{C}_{1}(t)=\frac{2}{3 \pi}\left(\frac{\mu^{2}}{e^{C} m^{2}}\right)^{u} & {\left[\frac{(2-u)\left(1+2 u+3 u^{2}\right)}{u(1+u)(1+2 u)} \frac{\Gamma(1-2 u) \Gamma(1+u)}{\Gamma(3-u)}\right.} \\
& \left.-\frac{1}{1+u} \frac{\Gamma(1-u) \Gamma\left(\frac{1+u}{2}\right)}{\Gamma\left(\frac{3-u}{2}\right)}\right]
\end{aligned}
$$

The Borel transform of the vertex correction in Fig. 5b is obtained by replacing $\gamma^{\mu}$ by

$$
i \frac{16 \pi}{3}\left(\frac{\mu^{2}}{e^{C}}\right)^{u} \int_{l} \frac{1}{\left(-l^{2}\right)^{1+u}} \gamma^{\alpha} \frac{1}{\not-\not \gamma-m} \gamma^{\mu} \frac{1}{\not+\not p-m} \gamma_{\alpha} .
$$

Using Feynman parameters to evaluate the integrals for the four vertex correction diagrams, we find that their contribution to $\widetilde{C}^{\gamma \gamma}$ is

$$
\begin{aligned}
\widetilde{C}_{2}(t)= & \frac{2}{3 \pi}\left(\frac{\mu^{2}}{e^{C} m^{2}}\right)^{u}\left[\frac{2(1-2 u)}{u^{2}} \frac{\Gamma(1-2 u) \Gamma(1+u)}{\Gamma(3-u)}\right. \\
& \left.+\frac{1}{u} \frac{\Gamma(1-u) \Gamma\left(\frac{1+u}{2}\right)}{\Gamma\left(\frac{3-u}{2}\right)}-\frac{1}{u^{2}} \frac{\Gamma(2-u) \Gamma\left(\frac{2+u}{2}\right)}{2 \Gamma\left(\frac{4-u}{2}\right)}\right] .
\end{aligned}
$$

The Borel transform of the self-energy correction for the internal quark line in Fig. 5d is given by (60) with $p$ replaced by $-r$. With a straightforward calculation, we obtain

$$
\widetilde{\Sigma}_{f}(-\not h, t)=-\frac{1}{3 \pi}\left(\frac{\mu^{2}}{e^{C} m^{2}}\right)^{u}\left[\not p \frac{\Gamma(-u) \Gamma\left(\frac{2+u}{2}\right)}{\Gamma\left(\frac{4-u}{2}\right)}+2 m \frac{\Gamma(-u) \Gamma\left(\frac{1+u}{2}\right)}{\Gamma\left(\frac{3-u}{2}\right)}\right] .
$$

The diagram in Fig. 5e has the mass counterterm $\delta \tilde{m}$ inserted into the internal quark line. Adding the contributions to $\widetilde{C}^{\gamma \gamma}$ from Figs. $5 \mathrm{~d}$ and $5 \mathrm{e}$, we obtain

$\widetilde{C}_{3}(t)=\frac{2}{3 \pi}\left(\frac{\mu^{2}}{e^{C} m^{2}}\right)^{u}\left[-\frac{1}{u} \frac{2 \Gamma(1-u) \Gamma\left(\frac{1+u}{2}\right)}{\Gamma\left(\frac{3-u}{2}\right)}+\frac{3(1-u)}{u} \frac{\Gamma(1-2 u) \Gamma(1+u)}{\Gamma(3-u)}\right]$.

Adding up (99), (101), (103), and (96), the Borel transform of the short-distance coefficient for the process $\eta_{c} \rightarrow \gamma \gamma$ is 


$$
\begin{aligned}
\tilde{C}^{\gamma \gamma}(t)=\frac{2}{3 \pi}\left(\frac{\mu^{2}}{e^{C} m^{2}}\right)^{u} & {\left[\frac{2\left(1+2 u-4 u^{2}-5 u^{3}+3 u^{5}\right)}{u^{2}(1+u)(1+2 u)} \frac{\Gamma(1-2 u) \Gamma(1+u)}{\Gamma(3-u)}\right.} \\
& \left.-\frac{1}{1+u} \frac{\Gamma(1-u) \Gamma\left(\frac{1+u}{2}\right)}{\Gamma\left(\frac{3-u}{2}\right)}-\frac{1}{u^{2}} \frac{\Gamma(1-u) \Gamma\left(\frac{2+u}{2}\right)}{\Gamma\left(\frac{4-u}{2}\right)}\right] .
\end{aligned}
$$

While individual terms in (104) have a double pole at $u=0$, there are cancellations that make the expression analytic at $u=0$. From eq. (104), we see that there are ultraviolet renormalons at the negative integers $u=-1,-2, \cdots$. There are infrared renormalons at the even and odd positive half-integers $u=\frac{1}{2}, 1, \frac{3}{2}, 2, \cdots$. Finally, there is the NRQCD ultraviolet renormalon at $u=-\frac{1}{2}$.

To obtain the power series expansion of $C^{\gamma \gamma}\left(\alpha_{s}\right)$, we expand (104) in powers of $u$ and carry out the inverse Borel transform by substituting $t^{n-1} \rightarrow(n-1) ! \alpha_{s}^{n}$. The first two terms in the expansion are

$$
C^{\gamma \gamma}\left(\alpha_{s}\right)=\frac{\pi^{2}-20}{6} \frac{\alpha_{s}(\mu)}{\pi}+\left(5.073+\frac{\pi^{2}-20}{3} \log \frac{\mu}{e^{C / 2} m}\right) \frac{\beta_{0} \alpha_{s}^{2}}{\pi} .
$$

The coefficient of $\alpha_{s}$ was first calculated by Barbieri et al. [22]. From the vanishing of the coefficient of $\beta_{0} \alpha_{s}^{2}$, we identify the BLM scale for this process to be $\mu=4.49 e^{C / 2} \mathrm{~m}$.

\section{RENORMALON SINGULARITIES IN QUARKONIUM ANNIHILATION DECAYS}

We have calculated the Borel transforms of the short-distance coefficients $C^{\Gamma}\left(\alpha_{s}\right)$ for $\Gamma=V, A, T, P$, and $\gamma \gamma$ in the large- $N_{f}$ limit. They have renormalon singularities in the form of poles on the real axis of the Borel parameter $t=u / \beta_{0}$. There are ultraviolet renormalons at the points $u=-1,-2, \cdots$, infrared renormalons at $\frac{1}{2}, \frac{3}{2}, 2, \frac{5}{2}, 3, \cdots$, and a NRQCD ultraviolet renormalon at $u=-\frac{1}{2}$. Each renormalon generates $n$ ! growth of the coefficients in the perturbation series, as shown in (48). The asymptotic behavior of the coefficients is dominated by the renormalons closest to the origin at $u= \pm \frac{1}{2}$. The contributions from a higher renormalon at $u=k / 2$ are suppressed by $k^{-n}$. In this section, we study in detail the dominant renormalons at $u=+\frac{1}{2}$ and $u=-\frac{1}{2}$. We show that the Borel-resummable renormalon at $u=-\frac{1}{2}$ arises from the cancellation of the Coulomb singularity in the short-distance coefficients and is universal in the sense that its residue is identical for all color-singlet $S$-wave decay processes. We show that the ambiguities from the renormalon at $u=\frac{1}{2}$ can be absorbed into the nonperturbative NRQCD matrix elements that contribute through relative order $v^{2}$. We also deduce the residues of the renormalons at $u= \pm \frac{1}{2}$ for the decay $J / \psi \rightarrow \gamma \gamma \gamma$.

\section{A. Universality of the $u=-\frac{1}{2}$ renormalon}

The Borel transforms of the short-distance coefficients $C^{\Gamma}\left(\alpha_{s}\right)$ are given in (84)-(87) for $\Gamma=V, A, T, P$ and in (104) for $\Gamma=\gamma \gamma$. By examining these expressions, we see that they 
all have a renormalon at $u=-\frac{1}{2}$ and that the residues of the poles are identical for all $\Gamma$. Thus they can all be written in the form

$$
\widetilde{C}^{\Gamma}(t)=\frac{1}{1+2 \beta_{0} t}\left(-\frac{8}{3 \pi} \frac{e^{C / 2} m}{\mu}\right)+\widetilde{B}^{\Gamma}(t)
$$

where $\widetilde{B}^{\Gamma}(t)$ is analytic at $\beta_{0} t=-\frac{1}{2}$. Since the pole at $u=-\frac{1}{2}$ is located on the negative real axis, the contribution of this renormalon to $C^{\Gamma}\left(\alpha_{s}\right)$ is Borel resummable. If $B^{\Gamma}\left(\alpha_{s}\right)$ is the inverse Borel transform of $\widetilde{B}^{\Gamma}(t)$, then the inverse Borel transform of (106) is

$$
C^{\Gamma}\left(\alpha_{s}\right)=-\frac{8}{3 \pi} \frac{e^{C / 2} m}{\mu} \alpha_{s} \int_{0}^{\infty} d x \frac{e^{-x}}{1+2 \beta_{0} \alpha_{s} x}+B^{\Gamma}\left(\alpha_{s}\right) .
$$

We have suppressed the $\mu$-dependence of the coupling constant $\alpha_{s}(\mu)$. For sufficiently small values of $\alpha_{s}$, the integral is approximately 1 . However its power series expansion in $\alpha_{s}$ is an alternating series whose coefficients diverge like $n$ !.

We found that the residues of the renormalon at $u=-\frac{1}{2}$ are the same for all processes that we calculated. We will show that this result is general, that the residue is identical for all color-singlet $S$-wave annihilation decay processes. The appearance of this renormalon can be traced back to a remnant of the cancellation of the Coulomb singularity in the short-distance coefficients.

Any $S$-wave charmonium decay proceeds through the annihilation of the $c \bar{c}$ pair. The $c \bar{c}$ annihilation amplitude at tree level defines a vertex $\Gamma(p, \bar{p})$, which depends on the 3-momenta of the $c$ and $\bar{c}$. The scale of the momentum dependence is $m$. The vertex can therefore be approximated by a momentum independent vertex when $c$ and $\bar{c}$ have 3-momenta that are small compared to $m$. The QCD radiative corrections to the annihilation amplitude include the diagram in Fig. 4 in which a virtual gluon is exchanged between the $c$ and $\bar{c}$. If the relative momentum between the $c$ and $\bar{c}$ is zero, there is a pinch singularity in the loop integral. This is the Coulomb singularity. If the $c$ and $\bar{c}$ have a small relative momentum $m v$, the singularity appears as a $1 / v$ term in the annihilation amplitudes. Since NRQCD by construction matches full QCD at momenta small compared to $m$, this Coulomb singularity is the same in the two theories. It therefore cancels in the short-distance coefficients. However, the cancellation leaves a finite remainder. If we insert a chain of $n$ bubble diagrams, into the propagator of the exchanged gluons, there is still a cancellation of the Coulomb singularity, but the remainder grows like $n$ !. These remainders form an alternating series. which is Borel resummable. This series produces the renormalon in the Borel transform at $u=-\frac{1}{2}$.

We proceed to calculate the Borel transform of the sum of diagrams in Fig. 4 in the full theory. The expression is identical to $(\sqrt{79})$, except that $\Gamma$ is replaced by a momentumdependent vertex $\Gamma(p-l, p+l)$ :

$$
\begin{aligned}
i 4 \pi\left(\frac{\mu^{2}}{e^{C}}\right)^{u} & \int_{l} \frac{1}{\left(-l^{2}-i \epsilon\right)^{1+u}} \\
& \times \bar{v}(p)\left[\gamma^{\nu} \frac{\not-\not p+m}{l^{2}-2 l \cdot p+i \epsilon} T^{a} \Gamma(p-l, p+l) T^{a} \frac{\not+\not p+m}{l^{2}+2 l \cdot p+i \epsilon} \gamma_{\nu}\right] u(p) .
\end{aligned}
$$

Since the Coulomb singularity comes from the region of $l \ll m$, the $l$ terms in the numerator and in the vertex $\Gamma$ can be neglected. Using $\not p u=m u$ and $\bar{v} \not p=-m \bar{v}$, the above equation can be reduced to 


$$
\begin{aligned}
-i 16 \pi m^{2}\left(\frac{\mu^{2}}{e^{C}}\right)^{u} & \bar{v}(p) T^{a} \Gamma(p, p) T^{a} u(p) \\
& \times \int_{l} \frac{1}{\left(-l^{2}-i \epsilon\right)^{1+u}} \frac{1}{l^{2}-2 m l_{0}+i \epsilon} \frac{1}{l^{2}+2 m l_{0}+i \epsilon} .
\end{aligned}
$$

If the annihilation vertex $\Gamma$ is a color-singlet, the factor $\bar{v} T^{a} \Gamma T^{a} u$ reduces to $(4 / 3) \bar{v} \Gamma u$. If $\Gamma$ is a color-octet vertex, the factor $4 / 3$ is replaced by $-1 / 6$.

To evaluate this integral, we first integrate over $l_{0}$ by using the contour integral method. Closing the contour in the upper-half plane, the Coulomb singularity appears in the contribution from the pole at $l_{0}^{*}=-m+\sqrt{m^{2}+l^{2}}+i \epsilon$. Keeping only this term, (109) reduces in the case of a color-singlet annihilation vertex to

$$
\frac{16 \pi m^{2}}{3}\left(\frac{\mu^{2}}{e^{C}}\right)^{u} \bar{v}(p) \Gamma(p, p) u(p) \int \frac{d^{3} \mathbf{l}}{(2 \pi)^{3}} \frac{1}{\left(2 m \sqrt{m^{2}+\mathbf{l}^{2}}-2 m^{2}\right)^{2+u}} \frac{1}{\sqrt{m^{2}+\mathbf{l}^{2}}} .
$$

The first factor in the integrand reduces to $1 /\left(\mathbf{l}^{2}\right)^{2+u}$ for small $\mathbf{l}$. It is easy to see that if (110) is expanded as a power series in $u$, all the coefficients have a linear infrared divergence coming from the small $\mathbf{l}$ region. This just corresponds to the Coulomb singularity.

We next consider the Borel transform of the sum of the diagrams in Fig. 4 in NRQCD. Since there are no momentum scales in the diagrams and since it must match (110) at low momenta, the result must be

$$
\frac{16 \pi m}{3}\left(\frac{\mu^{2}}{e^{C}}\right)^{u} \bar{v}(p) \Gamma(p, p) u(p) \int \frac{d^{3} \mathbf{l}}{(2 \pi)^{3}} \frac{1}{\left(\mathbf{l}^{2}\right)^{2+u}} .
$$

The contribution of the Coulomb singularity to the short-distance coefficient $\widetilde{C}^{\Gamma}(t)$ is obtained by subtracting (111) from (110) and removing the factor $\bar{v} \Gamma u$. The Coulomb singularity cancels, so the power series expansion of $\widetilde{C}^{\Gamma}(t)$ has finite coefficients. However, the integral in (111) has a power ultraviolet divergence when $u>-\frac{1}{2}$, and this produces a pole in $\widetilde{C}^{\Gamma}(t)$ at $u=-\frac{1}{2}$. The pole can be isolated by imposing a cutoff $|\mathbf{l}|>\kappa$ on the integral:

$$
\widetilde{C}^{\Gamma}(t) \sim-\frac{16 \pi m}{3}\left(\frac{\mu^{2}}{e^{C}}\right)^{u} \int_{|\mathbf{1}|>\kappa} \frac{d^{3} \mathbf{l}}{(2 \pi)^{3}} \frac{1}{\left(\mathbf{l}^{2}\right)^{2+u}} .
$$

Evaluating the integral, we find that the residue of the pole is identical to that in (106).

It is easy to see that the renormalon at $u=-\frac{1}{2}$ and its residue are gauge invariant. If we had used the gluon propagator (55) to carry out the calculation in a general covariant gauge, there would be additional terms in (108) with $\gamma_{\nu}$ replaced by $\ell$. Making the decomposition $\gamma=(\not p-m)-(\not p-\not \gamma-m)$, the first term vanishes due to the equation of motion for $u(p)$, while the second term cancels the quark propagator. This removes the pinch singularity, and therefore these terms have no pole at $u=-\frac{1}{2}$.

The residue of the $u=-\frac{1}{2}$ renormalon is universal for all color-singlet $S$-wave quarkonium annihilation decays. The universality follows from the fact that in the low-momentum region of the loop integral for the QCD amplitude (108), the $c \bar{c}$ annihilation amplitude can be approximated by a vertex $\Gamma(p, p)$ that is independent of the loop momentum and nonzero for $S$-wave annihilation. The low-momentum regions of the QCD loop integral and the 
corresponding NRQCD loop integral must match. But there are no momentum scales in the NRQCD loop integral, and therefore its ultraviolet behavior is also fixed. The residue of the ultraviolet NRQCD renormalon at $u=-\frac{1}{2}$ depends only on whether the annihilation amplitude $\Gamma(p, p)$ is color-singlet or color-octet. Note that this renormalon is generated only by loop diagrams in which a gluon is exchanged between the $c$ and $\bar{c}$. Other loop diagrams, such as quark self-energy diagrams and the diagrams in which a gluon connects the $c$ or $\bar{c}$ line to the annihilation vertex, give no contributions to the renormalon simply because there is no pinch singularity in the QCD diagrams.

\section{B. Cancellation of the $u=\frac{1}{2}$ renormalon ambiguities}

The Borel transforms $\widetilde{C}^{\Gamma}(t)$ in (84)-(87) and (104) have an infrared renormalon at $u=$ $+\frac{1}{2}$. They can therefore be written in the form

$$
\widetilde{C}^{\Gamma}(t)=\frac{a^{\Gamma}}{1-2 \beta_{0} t}+\widetilde{A}^{\Gamma}(t),
$$

where $\widetilde{A}^{\Gamma}(t)$ is analytic at $\beta_{0} t=\frac{1}{2}$ and the residues of the poles are

$$
\begin{aligned}
a^{V} & =-\frac{11}{9 \pi} \frac{\mu}{e^{C / 2} m} \\
a^{A} & =-\frac{1}{3 \pi} \frac{\mu}{e^{C / 2} m} \\
a^{T} & =-\frac{5}{3 \pi} \frac{\mu}{e^{C / 2} m}, \\
a^{P} & =\frac{1}{\pi} \frac{\mu}{e^{C / 2} m}, \\
a^{\gamma \gamma} & =\frac{5}{9 \pi} \frac{\mu}{e^{C / 2} m} .
\end{aligned}
$$

Since the pole at $u=\frac{1}{2}$ is located on the positive real axis, the inverse Borel transform is not unique. Taking the principal value of the integral in (47) is only one of the possible prescriptions. If $A^{\Gamma}\left(\alpha_{s}\right)$ is the inverse Borel transform of $\widetilde{A}^{\Gamma}(t)$, the general expression for the inverse Borel transform of (113) is

$$
C^{\Gamma}\left(\alpha_{s}\right)=a^{\Gamma} \alpha_{s} \mathrm{P} \int_{0}^{\infty} d x \frac{e^{-x}}{1-2 \beta_{0} \alpha_{s} x}+A^{\Gamma}\left(\alpha_{s}\right)-K \frac{\pi a^{\Gamma}}{\beta_{0}} e^{-1 /\left(2 \beta_{0} \alpha_{s}\right)}
$$

where $\mathrm{P} \int$ denotes the principal value integral, $K$ is an arbitrary constant, and $\alpha_{s}=\alpha_{s}(\mu)$. The last term in (119) represents the ambiguity in the inverse Borel transform, which is proportional to the residue of the pole in the integrand. Using the one-loop expression (50) for the running coupling constant $\alpha_{s}(\mu)$ and the fact that $a^{\Gamma}$ includes a factor of $\mu / m$, we see that the ambiguity in (119) is proportional to $\Lambda_{Q C D} / \mathrm{m}$. This ambiguity arises because summing the series of loop diagrams with bubble chains inserted into the gluon propagator is equivalent to replacing the strong coupling constant by a running coupling constant at the scale of the gluon momentum. However, the perturbative running coupling constant has a Landau pole at the momentum scale $\Lambda_{\mathrm{QCD}}$. This pole appears in the integration region 
and therefore gives rise to an ambiguity in the loop integral. The renormalon ambiguity implies that the short-distance coefficients defined by minimal subtraction necessarily have some sensitivity to nonperturbative effects involving the energy scale $\Lambda_{\mathrm{QCD}}$.

The infrared renormalons give ambiguities in the short-distance coefficients in NRQCD factorization formulas. The decay rates for $J / \psi \rightarrow e^{+} e^{-}$and $\eta_{c} \rightarrow \gamma \gamma$ and the matrix elements in (75)-(77) are physical quantities, so they cannot have any ambiguities. The NRQCD factorization formulas simply represent the separation of scales. All effects of momentum scales $m$ and larger are included in the short-distance coefficients, while effects of lower scales, such as $m v, m v^{2}$, and $\Lambda_{\mathrm{QCD}}$, are absorbed into the NRQCD matrix elements. Since infrared renormalons arise from small momentum scales, any ambiguities in the coefficients due to infrared renormalons must be compensated by corresponding ambiguities in the nonperturbative matrix elements. Thus it must be possible to consistently absorb the ambiguities proportional to $K$ in (119) for $\Gamma=V, A, T, P$ and $\gamma \gamma$ into various NRQCD matrix elements.

The ambiguities in a coefficient from an infrared renormalon at $u=k / 2$, where $k$ is an integer, is proportional to $\left(\Lambda_{\mathrm{QCD}} / m\right)^{k}$. Since the NRQCD matrix elements involve several different scales, it is not immediately obvious which matrix element an ambiguity proportional to $\Lambda_{\mathrm{QCD}}^{k}$ should be absorbed into. We will make a simple hypothesis that is consistent with the velocity-scaling rules of NRQCD and verify that the ambiguities due to the $u=+\frac{1}{2}$ renormalon are consistent with this hypothesis. Our hypothesis is that ambiguities proportional to $\Lambda_{\mathrm{QCD}}^{k}$ can be absorbed into NRQCD matrix elements that are suppressed by $v^{2 k}$ or less. If our hypothesis is correct, ambiguities due to the $u=+\frac{1}{2}$ renormalon should be absorbed into the leading order matrix elements and those suppressed by $v^{2}$. In the case of the $J / \psi$, these matrix elements are $\left\langle 0\left|\chi^{\dagger} \sigma^{i} \psi\right| J / \psi\right\rangle$ and $\left\langle 0\left|\chi^{\dagger} \mathbf{D}^{2} \sigma^{i} \psi\right| J / \psi\right\rangle$. In the case of $\eta_{c}$, they are $\left\langle 0\left|\chi^{\dagger} \psi\right| \eta_{c}\right\rangle$ and $\left\langle 0\left|\chi^{\dagger} \mathbf{D}^{2} \psi\right| \eta_{c}\right\rangle$. Because of the spin-symmetry relation (7), there are only three independent matrix elements that are suppressed by $v^{2}$ or less. It is convenient to introduce the following shorthand notation for these three independent matrix elements:

$$
\begin{aligned}
\left\langle\mathcal{K}_{\psi}\right\rangle & \equiv \boldsymbol{\epsilon} \cdot\left\langle 0\left|\chi^{\dagger} \boldsymbol{\sigma} \psi\right| J / \psi(\boldsymbol{\epsilon})\right\rangle \\
\left\langle\mathcal{K}_{\eta}\right\rangle & \equiv\left\langle 0\left|\chi^{\dagger} \psi\right| \eta_{c}\right\rangle \\
\left\langle\mathcal{K}_{D^{2}}\right\rangle & \equiv \frac{1}{m^{2}}\left\langle 0\left|\chi^{\dagger} \mathbf{D}^{2} \psi\right| \eta_{c}\right\rangle
\end{aligned}
$$

We first consider the cancellation of the renormalon ambiguities in the QCD matrix elements in (74)-(77). The coefficients of the matrix element $\left\langle\mathcal{K}_{D^{2}}\right\rangle$ in these factorization formulas are given in (14) and (42)-(44). The coefficients $C^{\Gamma}$ are functions of $\beta_{0} \alpha_{s}$ divided by $\beta_{0}$ and are therefore suppressed by $1 / N_{f}$ in the large- $N_{f}$ limit. Since the ambiguities $\Delta C^{\Gamma}$ in the coefficients are suppressed by $1 / N_{f}$, the ambiguities $\Delta\left\langle\mathcal{K}_{\psi}\right\rangle, \Delta\left\langle\mathcal{K}_{\eta}\right\rangle$, and $\Delta\left\langle\mathcal{K}_{D^{2}}\right\rangle$ in the matrix elements must also be suppressed by $1 / N_{f}$. The cancellation conditions for the renormalon ambiguities at leading order in $1 / N_{f}$ are therefore linear relations:

$$
\begin{aligned}
\Delta C^{V}\left\langle\mathcal{K}_{\psi}\right\rangle+\Delta\left\langle\mathcal{K}_{\psi}\right\rangle+\frac{1}{6} \Delta\left\langle\mathcal{K}_{D^{2}}\right\rangle & =0, \\
\Delta C^{A}\left\langle\mathcal{K}_{\eta}\right\rangle+\Delta\left\langle\mathcal{K}_{\eta}\right\rangle+\frac{1}{2} \Delta\left\langle\mathcal{K}_{D^{2}}\right\rangle & =0, \\
\Delta C^{T}\left\langle\mathcal{K}_{\psi}\right\rangle+\Delta\left\langle\mathcal{K}_{\psi}\right\rangle+\frac{1}{3} \Delta\left\langle\mathcal{K}_{D^{2}}\right\rangle & =0,
\end{aligned}
$$




$$
\Delta C^{P}\left\langle\mathcal{K}_{\eta}\right\rangle+\Delta\left\langle\mathcal{K}_{\eta}\right\rangle=0
$$

From (119), the ambiguities in the coefficients are

$$
\Delta C^{\Gamma}=-K \frac{\pi a^{\Gamma}}{\beta_{0}} \frac{\Lambda_{\mathrm{QCD}}}{\mu},
$$

with the coefficients $a^{\Gamma}$ given by (114)-(117). The cancellation conditions (123)-(126) are four equations for the three unknown ambiguities in the matrix elements, which provides a consistency check. The solutions are

$$
\begin{aligned}
\Delta\left\langle\mathcal{K}_{\psi}\right\rangle & =-K \frac{7}{9 \beta_{0}} \frac{\Lambda_{\mathrm{QCD}}}{e^{C / 2} m}\left\langle\mathcal{K}_{\psi}\right\rangle, \\
\Delta\left\langle\mathcal{K}_{\eta}\right\rangle & =K \frac{1}{\beta_{0}} \frac{\Lambda_{\mathrm{QCD}}}{e^{C / 2} m}\left\langle\mathcal{K}_{\eta}\right\rangle, \\
\Delta\left\langle\mathcal{K}_{D^{2}}\right\rangle & =-K \frac{8}{3 \beta_{0}} \frac{\Lambda_{\mathrm{QCD}}}{e^{C / 2} m}\left\langle\mathcal{K}_{\eta}\right\rangle .
\end{aligned}
$$

These results can be verified by perturbative calculations of the large- $N_{f}$ limits of the Borel transforms of the NRQCD matrix elements.

Now we examine the renormalon cancellations for the process $\eta_{c} \rightarrow \gamma \gamma$. From (32) and (91), the factorization formula for the $T$-matrix element can be written in the form

$$
\mathcal{T}_{\eta_{c} \rightarrow \gamma \gamma}=-i \frac{2 Q^{2} e^{2}}{M_{\eta_{c}}} \boldsymbol{\epsilon}_{1} \times \boldsymbol{\epsilon}_{2} \cdot \hat{\mathbf{r}}\left[\left(1+C^{\gamma \gamma}\right)\left\langle\mathcal{K}_{\eta}\right\rangle+\frac{1}{6}\left\langle\mathcal{K}_{D^{2}}\right\rangle\right]
$$

We have used the identity (34) to eliminate the pole mass $m_{\text {pole }}$ in the prefactor in favor of the meson mass $M_{\eta_{c}}$. The cancellation condition for the ambiguities from the renormalon at $u=\frac{1}{2}$ is

$$
\Delta C^{\gamma \gamma}\left\langle\mathcal{K}_{\eta}\right\rangle+\Delta\left\langle\mathcal{K}_{\eta}\right\rangle+\frac{1}{6} \Delta\left\langle\mathcal{K}_{D^{2}}\right\rangle=0
$$

Using the value of $a^{\gamma \gamma}$ in (118) and the values of $\Delta\left\langle\mathcal{K}_{\eta}\right\rangle$ and $\Delta\left\langle\mathcal{K}_{D^{2}}\right\rangle$ given in (129) and (130), we see that (132) is satisfied.

We can use the results given above to verify the cancellation of renormalon ambiguities in the Gremm-Kapustin identity, which is given at tree level in (17). The correct generalization to higher order in $\alpha_{s}$ is to replace $m$ by $m_{\text {pole }}$. The analogous identity for the matrix elements of $\eta_{c}$ is

$$
\left\langle 0\left|\chi^{\dagger} \mathbf{D}^{2} \psi\right| \eta_{c}\right\rangle=-m_{\text {pole }}\left(M_{\eta_{c}}-2 m_{\text {pole }}\right)\left\langle 0\left|\chi^{\dagger} \psi\right| \eta_{c}\right\rangle\left(1+O\left(v^{2}\right)\right) .
$$

From the expression (72) for the Borel transform of $m_{\text {pole }}$, we find that the ambiguity due to the renormalon at $u=+\frac{1}{2}$ is

$$
\Delta m_{\text {pole }}=-K \frac{4}{3 \beta_{0}} e^{-C / 2} \Lambda_{\mathrm{QCD}} .
$$

The leading contribution to the ambiguity on the right side of (133) comes from the factor $M_{\eta_{c}}-2 m_{\text {pole }}$. The ambiguities from the factors on the right side of (133) are suppressed by $v^{2}$ from the factor $M_{\eta_{c}}-2 m_{\text {pole }}$. Thus the renormalon cancellation condition is

$$
\Delta\left\langle\mathcal{K}_{D^{2}}\right\rangle=2 \frac{\Delta m_{\text {pole }}}{m}\left\langle\mathcal{K}_{\eta}\right\rangle
$$

Using (130) and (134), we see that this is satisfied. 


\section{Leading Renormalons for $J / \psi \rightarrow \gamma \gamma \gamma$}

The one-loop QCD correction to the short-distance coefficient for the process $J / \psi \rightarrow \gamma \gamma \gamma$ has been calculated by Mackenzie and Lepage [23]. The correction to the decay rate is a multiplicative factor $1-12.6 \alpha_{s} / \pi$, which gives a correction of about $-140 \%$ if we take the coupling constant to be $\alpha_{s}(m) \approx 0.35$. Since it is the leading order correction, the coefficient of $\alpha_{s}$ is independent of the renormalization scale and the renormalization scheme. The enormous radiative correction raises questions about the origin of the large coefficient of $\alpha_{s}$ and about the convergence of the perturbation series. Some insights can be gained by studying the renormalon singularities for this process. As shown above, the large order behavior of the short-distance coefficient is governed by the $u= \pm \frac{1}{2}$ renormalons. We know the residue of the $u=-\frac{1}{2}$ renormalon in the large $N_{f}$ limit, since it is universal for all $S$ -

wave annihilation decay processes. The residue of the $u=\frac{1}{2}$ renormalon can be determined by requiring the cancellation of the renormalon ambiguities in the decay width. Knowing the residues of the leading renormalons, we can estimate the asymptotic behavior of the perturbation series.

The decay width for $J / \psi \rightarrow \gamma \gamma \gamma$, with the coefficient of the first relativistic correction calculated to leading order, is

$$
\begin{aligned}
\Gamma(J / \psi \rightarrow \gamma \gamma \gamma)=\frac{16\left(\pi^{2}-9\right) Q^{6} \alpha^{3}}{9 M_{J / \psi}^{3}} \mid\left(1+C^{3 \gamma}\left(\alpha_{s}\right)\right)\left\langle 0\left|\chi^{\dagger} \boldsymbol{\sigma} \psi\right| J / \psi\right\rangle \\
+\left.\frac{7 \pi^{2}-24}{24\left(\pi^{2}-9\right)} \frac{1}{m^{2}}\left\langle 0\left|\chi^{\dagger} \mathbf{D}^{2} \boldsymbol{\sigma} \psi\right| J / \psi\right\rangle\right|^{2} .
\end{aligned}
$$

The coefficient of the matrix element $\left\langle 0\left|\chi^{\dagger} \mathbf{D}^{2} \boldsymbol{\sigma} \psi\right| J / \psi\right\rangle$ was first calculated by Keung and Muzinich in numerical form [24] and by Schuler in analytic form [25]. The condition for the cancellation of the $u=\frac{1}{2}$ renormalon ambiguities in $\Gamma(J / \psi \rightarrow \gamma \gamma \gamma)$ reads:

$$
\Delta C^{3 \gamma}\left\langle\mathcal{K}_{\psi}\right\rangle+\Delta\left\langle\mathcal{K}_{\psi}\right\rangle+\frac{7 \pi^{2}-24}{24\left(\pi^{2}-9\right)} \Delta\left\langle\mathcal{K}_{D^{2}}\right\rangle=0
$$

Using the values of $\Delta\left\langle\mathcal{K}_{\psi}\right\rangle$ and $\Delta\left\langle\mathcal{K}_{D^{2}}\right\rangle$ determined in (128) and (130), we obtain

$$
\Delta C^{3 \gamma}=K \frac{14 \pi^{2}-87}{9\left(\pi^{2}-9\right) \beta_{0}} \frac{\Lambda_{\mathrm{QCD}}}{e^{C / 2} m} .
$$

Comparing with (127), we can read off the residue $a^{3 \gamma}$ of the $u=\frac{1}{2}$ renormalon, which is defined by (113):

$$
a^{3 \gamma}=-\frac{14 \pi^{2}-87}{9\left(\pi^{2}-9\right) \pi} \frac{\mu}{e^{C / 2} m} .
$$

This residue is rather large. It differs from the residues $a^{V}$ and $a^{\gamma \gamma}$ for the decays $J / \psi \rightarrow e^{+} e^{-}$ and $\eta_{c} \rightarrow \gamma \gamma$ by factors of 5.4 and -11.8 , respectively. The large one-loop radiative correction may be related to the large residue of the $u=\frac{1}{2}$ renormalon. 


\section{ESTIMATES OF HIGHER ORDER RADIATIVE CORRECTIONS}

One of the problems encountered in phenomenological applications of the NRQCD factorization formalism is that the one-loop corrections to the short-distance coefficients for several decay rates are uncomfortably large. This raises questions about the convergence of the QCD perturbation series. The calculations of the short-distance coefficients in the large- $N_{f}$ limit can shed some light on the origin of the large radiative corrections. They can also be used to provide simple estimates of higher order radiative corrections. It has been found empirically that if we take the coefficients of the perturbation series in the large- $N_{f}$ limit and make the shift $N_{f} \rightarrow N_{f}-33 / 2$, we obtain reasonable approximations to the higher order coefficients in cases where they are known exactly [7]. This prescription, which has been called "naive nonabelianization", puts the Borel singularities at the correct points on the $t$-axis. We will use this prescription to give simple estimates of higher order corrections in the perturbation series for $C^{V}\left(\alpha_{s}\right), C^{\gamma \gamma}\left(\alpha_{s}\right)$, and $C^{3 \gamma}\left(\alpha_{s}\right)$.

The Borel transform $\widetilde{C}^{\Gamma}(t)$ for the short-distance coefficients in the decay rate for $J / \psi \rightarrow$ $e^{+} e^{-}$and $\eta_{c} \rightarrow \gamma \gamma$ are given in (84) and (104). To obtain the perturbation expansion for $C^{\Gamma}\left(\alpha_{s}\right)$ in the large- $N_{f}$ limit, we expand $\widetilde{C}^{\Gamma}(t)$ as a power series in $u$, and then invert the Borel transform by substituting $t^{n-1} \rightarrow(n-1) ! \alpha_{s}^{n}$. We set $\beta_{0}=\left(33-2 N_{f}\right) / 12 \pi$ and take $N_{f}=3$ for charmonium decays. We choose the $\overline{\mathrm{MS}}$ scheme by setting $C=-5 / 3$ and we take the scale of the running coupling constant to be $\mu=m$. The resulting perturbation series are

$$
\begin{gathered}
C^{V}\left(\alpha_{s}\right)=-2.7 a-5.5 a^{2}-150 a^{3}-870 a^{4}-38000 a^{5}-360000 a^{6}+\cdots, \\
C^{\gamma \gamma}\left(\alpha_{s}\right)=-1.7 a+5.1 a^{2}-25 a^{3}+1100 a^{4}-900 a^{5}+520000 a^{6}+\cdots .
\end{gathered}
$$

where $a=\alpha_{s}(m) / \pi$. We have kept only two significant figures in each of the coefficients.

The behavior of the perturbation series (140) and (141) can be understood by examining the contributions from the renormalon singularities at $u=\frac{1}{2}$ and $u=-\frac{1}{2}$. Asymptotically, these renormalons dominate the coefficients in the perturbation series, because the contribution to the coefficient of $\alpha_{s}^{n}$ from a higher renormalon at $u=k / 2$ is suppressed by $k^{-n}$. The

contributions to the perturbation series (140) and (141) from the renormalons at $u= \pm \frac{1}{2}$ are

$$
\begin{aligned}
C^{V}\left(\alpha_{s}\right) & \sim \frac{1}{2 \pi \beta_{0}} \sum_{n}\left[-\frac{11}{9} \frac{\mu}{e^{C / 2} m}+(-1)^{n} \frac{8}{3} \frac{e^{C / 2} m}{\mu}\right](n-1) !\left[2 \beta_{0} \alpha_{s}(\mu)\right]^{n}, \\
C^{\gamma \gamma}\left(\alpha_{s}\right) & \sim \frac{1}{2 \pi \beta_{0}} \sum_{n}\left[\frac{5}{9} \frac{\mu}{e^{C / 2} m}+(-1)^{n} \frac{8}{3} \frac{e^{C / 2} m}{\mu}\right](n-1) !\left[2 \beta_{0} \alpha_{s}(\mu)\right]^{n} .
\end{aligned}
$$

The alternating series in (142) and (143) are identical, since the $u=-\frac{1}{2}$ renormalon is universal for all $S$-wave annihilation decay processes. The same-sign series in (142) and (143) are proportional to the residues of the $u=+\frac{1}{2}$ renormalon. The relative importance of the two renormalons depends on the renormalization scale $\mu$. The $u=-\frac{1}{2}$ renormalon dominates if $\mu \ll m$ and the $u=+\frac{1}{2}$ renormalon dominates if $\mu \gg m$. The choice of $\mu$ that gives equal contributions from the two renormalons is $\mu=1.48 e^{C / 2} m$ for (142) and $\mu=2.19 e^{C / 2} m$ for $(143)$. In the $\overline{\mathrm{MS}}$ scheme, these scale are $0.64 m$ and $0.95 m$. These values suggest that $\mu=m$ is a reasonable value for the renormalization scale in the $\overline{\mathrm{MS}}$ scheme. For comparison, the corresponding BLM scales are $\mu=0.63 m$ and $\mu=1.95 m$, respectively. 
In the $\overline{\mathrm{MS}}$ scheme $\left(C=-\frac{5}{3}\right)$ with the scale $\mu=m$, the first few terms in the series (142) and (143) are

$$
\begin{aligned}
C^{V}\left(\alpha_{s}\right) & \sim-4.0 a-7.4 a^{2}-160 a^{3}-900 a^{4}-39000 a^{5}-370000 a^{6}+\cdots, \\
C^{\gamma \gamma}\left(\alpha_{s}\right) & \sim 0.12 a+11 a^{2}+4.8 a^{3}+1300 a^{4}+1200 a^{5}+540000 a^{6}+\cdots .
\end{aligned}
$$

Comparing (140) and (144), we see that the leading renormalons begin to dominate the large- $N_{f}$ coefficients of $C^{V}$ at the $\alpha_{s}^{3}$ term. Comparing (141) and (145), we see that the leading renormalons dominate the even coefficients of $C^{\gamma \gamma}$ beginning at the $\alpha_{s}^{4}$ term, but they do not dominate the odd coefficients until much higher order in $\alpha_{s}$. The reason for this is that the coefficient in square brackets in (143) is $1.28+(-1)^{n} 1.16$. The asymptotic coefficients for $C^{\gamma \gamma}$ are therefore positive and grow like $(n-1) ! \beta_{0}^{n-1}$. However, for odd $n$, there is a near cancellation between the contributions of the renormalons at $u= \pm \frac{1}{2}$ that delays the onset of the asymptotic behavior. At preasymptotic values of $n$, the odd coefficients may be dominated by the next higher renormalons at $u= \pm 1$.

We now consider the higher order radiative corrections to the decay rate for $J / \psi \rightarrow \gamma \gamma \gamma$. The large- $N_{f}$ limit of the short-distance coefficient $C^{3 \gamma}\left(\alpha_{s}\right)$ has not yet been calculated. Only the first term in the perturbation series is known [23]:

$$
C^{3 \gamma}\left(\alpha_{s}\right)=-6.3 a+\cdots
$$

However we have determined the residues of the $u= \pm \frac{1}{2}$ renormalons, and we can use that information to deduce the asymptotic behavior of the coefficients in the large- $N_{f}$ limit. The contributions from the $u=-\frac{1}{2}$ and $u=\frac{1}{2}$ renormalons are

$$
C^{3 \gamma}\left(\alpha_{s}\right) \sim \frac{1}{2 \pi \beta_{0}} \sum_{n}\left[-\frac{14 \pi^{2}-87}{9\left(\pi^{2}-9\right)} \frac{\mu}{e^{C / 2} m}+(-1)^{n} \frac{8}{3} \frac{e^{C / 2} m}{\mu}\right](n-1) !\left[2 \beta_{0} \alpha_{s}(\mu)\right]^{n} .
$$

In the $\overline{\mathrm{MS}}$ scheme $(C=-5 / 3)$ with $\mu=m$, the first few terms in the series are

$$
C^{3 \gamma}\left(\alpha_{s}\right) \sim-16 a-62 a^{2}-660 a^{3}-7600 a^{4}-160000 a^{5}-3100000 a^{6}+\cdots
$$

where $a=\alpha_{s}(m) / \pi$. The asymptotic coefficients are almost an order of magnitude large than those in (144) and (145). The large coefficients are caused by the large residue of the $u=+\frac{1}{2}$ renormalon.

One can attribute the large residue of the $u=+\frac{1}{2}$ renormalon in $C^{3 \gamma}\left(\alpha_{s}\right)$ to a poor choice of the renormalization scale $\mu$. The contribution from the $u=+\frac{1}{2}$ renormalon can be decreased at the expense of increasing the contribution from the $u=-\frac{1}{2}$ renormalon. The best balance between the two sources of $n$ ! growth is to choose $\mu$ so that the two renormalons contribute equally to the asymptotic coefficients. This prescription gives the rather small value $\mu=0.28 \mathrm{~m}$ for the scale. With this choice of scale, the even coefficients in (148) vanish and the odd coefficients are decreased in magnitude by about a factor of 2 . This scale is rather close to the BLM scale for the closely related process $J / \psi \rightarrow g g g$, which is $\mu=0.31 \mathrm{~m}$. This suggests that this may indeed be an appropriate choice for $\mu$ in the $\overline{\mathrm{MS}}$ scheme.

We now consider briefly the phenomenological implications of our calculations for charmonium decays, where the appropriate coupling constant is $\alpha_{s}(m) \approx 0.35$. The first terms in (140), (141), and (146) give the complete order- $\alpha_{s}$ corrections to the decay amplitudes. They 
represent radiative corrections of $-30 \%$ for $J / \psi \rightarrow e^{+} e^{-},-19 \%$ for $\eta_{c} \rightarrow \gamma \gamma$, and $-70 \%$ for $J / \psi \rightarrow \gamma \gamma \gamma$. The order- $\alpha_{s}^{2}$ correction is known only in the large- $N_{f}$ limit for $J / \psi \rightarrow e^{+} e^{-}$ and $\eta_{c} \rightarrow \gamma \gamma$, and it gives corrections of $-7 \%$ and $+6 \%$. For $J / \psi \rightarrow \gamma \gamma \gamma$, we only know the contribution to the order- $\alpha_{s}^{2}$ correction from the renormalons at $u= \pm \frac{1}{2}$, which give a correction of $-70 \%$. We conclude that the perturbation series for the amplitudes for the decays $J / \psi \rightarrow e^{+} e^{-}$and $\eta_{c} \rightarrow \gamma \gamma$ appear to be well-behaved through next-to-next-to-leading order in $\alpha_{s}$. However, the perturbation series for $J / \psi \rightarrow \gamma \gamma \gamma$ appears to be hopelessly divergent. One might be able to decrease the order- $\alpha_{s}^{2}$ correction by changing the renormalization scale to $\mu \approx m / 3$, but only at the cost of increasing the order- $\alpha_{s}$ correction to well over $100 \%$.

\section{CONCLUSION}

In this paper, we studied the large-order asymptotic behavior of the perturbation series for short-distance coefficients in the NRQCD factorization formalism. We calculated the Borel transforms of the short-distance coefficients for the electromagnetic decays $J / \psi \rightarrow$ $e^{+} e^{-}$and $\eta_{c} \rightarrow \gamma \gamma$ in the large- $N_{f}$ limit. We found that there exists a universal resummable renormalon associated with the cancellation of the Coulomb singularity in the short-distance coefficients. We verified that the ambiguities in the short-distance coefficients due to the first infrared renormalon are canceled by ambiguities in the long-distance matrix elements that contribute through relative order $v^{2}$. We used our calculation to make estimates of the higher order radiative corrections to the decay rates for $J / \psi \rightarrow e^{+} e^{-}, \eta_{c} \rightarrow \gamma \gamma$, and $J / \psi \rightarrow \gamma \gamma \gamma$. This work represents a first step toward studying the effects of renormalons in the NRQCD factorization formalism. We hope that further work in this direction can lead to an understanding of how to deal with the large radiative corrections encountered in some decay rates, such as $J / \psi \rightarrow \gamma \gamma \gamma$ and $J / \psi \rightarrow g g g$.

\section{ACKNOWLEDGMENTS}

This work was supported in part by the U.S. Department of Energy, Division of High Energy Physics, under Grant DE-FG02-91-ER40684. We acknowledge valuable discussions with G.T. Bodwin. 


\section{REFERENCES}

[1] G.T. Bodwin, E. Braaten, and G.P. Lepage, Phys. Rev. D 51, 1125 (1995).

[2] E. Braaten, in Particle Physics Phenomenology, edited by D. Chang et al. (World Scientific, Singapore, 1997) (hep-ph/9702225).

[3] G. 't Hooft, in The Whys of Subnuclear Physics, edited by A. Zichichi (Plenum, New York, 1978).

[4] A. Mueller, Nucl. Phys. B250, 327 (1980).

[5] M. Beneke, Nucl. Phys. B405, 424 (1993).

[6] M. Neubert, Nucl. Phys. B463, 511 (1996).

[7] P. Ball, M. Beneke, and V. M. Braun, Nucl. Phys. B452, 563 (1995).

[8] M. Beneke and V. M. Braun, Nucl. Phys. B426, 301 (1994).

[9] I.I. Bigi, M. Shifman, N.G. Uraltsev, and A.I. Vainshtein, Phys. Rev. D 50, 2234 (1994).

[10] M. Neubert and C.T. Sachrajda, Nucl. Phys. B438, 235 (1995).

[11] M. Luke, A. Manohar, and M.J. Savage, Phys. Rev. D 51, 4924 (1995).

[12] A. Petrelli, M. Cacciari, M. Greco, F. Maltoni, M.L. Mangano, CERN-TH-97-142 hepph/9707223).

[13] E. Braaten and Y.-Q. Chen, Phys. Rev. D 54, 3216 (1996); 55, 2693 (1997); 55, 7152 (1997).

[14] L.L. Foldy and S.A. Wouthuysen, Phys. Rev. 78, 29 (1950); S. Tani, Prog. Theor. Phys. 6, 267 (1951).

[15] I. Maksymyk (hep-ph/9710291).

[16] M. Gremm and A. Kapustin, Phys. Lett. 407B, 323 (1997).

[17] C. Cronstrom, Phys. Lett. 90B, 267(1980).

[18] A. Palanques-Mestre and P. Pascual, Commun. Math. Phys. 95, 277 (1984).

[19] D.J. Broadhurst, Z. Phys. C 58, 339 (1993).

[20] R. Barbieri, R. Gatto, R. Kögerler, and Z. Kunszt, Phys. Lett. 57B, 455 (1975); W. Celmaster, Phys. Rev. D 19, 1517 (1979).

[21] S. Brodsky, G.P. Lepage, and P. Mackenzie, Phys. Rev. D 28228 (1983).

[22] R. Barbieri, G. Curci, E. d'Emilio, and E. Remiddi, Nucl. Phys. B154, 535 (1979); K. Hagiwara, C.B. Kim, and T. Yoshino, Nucl. ibid. B177, 461 (1981).

[23] P. Mackenzie and G.P. Lepage, Phys. Rev. Lett. 47, 1244 (1981); P. Mackenzie and G.P. Lepage, in Perturbative Quantum Chromodynamics, edited by D.W. Duke and J.F. Owens (American Institute of Physics, New York, 1981).

[24] W.-Y. Keung and I.J. Muzinich, Phys. Rev. D 27, 1518 (1983).

[25] G. Schuler, CERN-TH-7170-94 (hep-ph/9403387). 


\section{FIGURES}

Fig. 1. Tree-level diagram for $c \bar{c} \rightarrow e^{+} e^{-}$.

Fig. 2. Tree-level diagrams for $c \bar{c} \rightarrow \gamma \gamma$.

Fig. 3. One-loop diagram for the heavy-quark self-energy, with a chain of light quark bubbles inserted into the gluon propagator.

Fig. 4. One-loop vertex correction for $c \bar{c} \rightarrow e^{+} e^{-}$, with a chain of light quark bubbles inserted into the gluon propagator.

Fig. 5. One-loop diagrams for $c \bar{c} \rightarrow \gamma \gamma$, with a chain of light quark bubbles inserted into the gluon propagator. For each diagram, there is a corresponding diagram with the photon lines interchanged. The blob in Figure 5e represents the mass counterterm. 


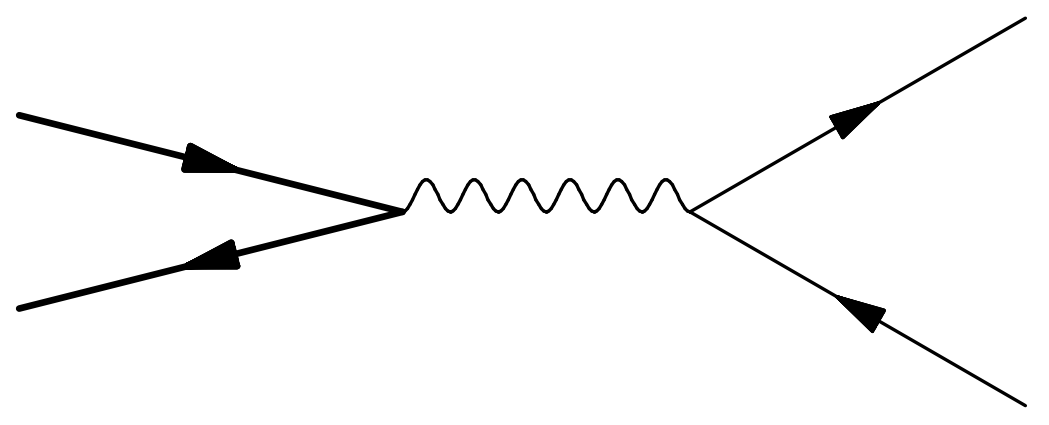

Figure 1
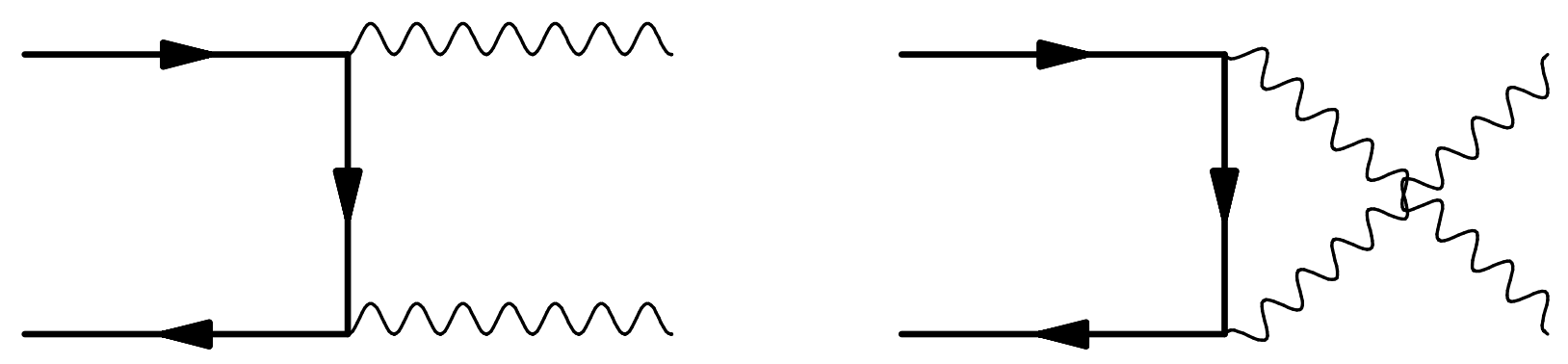

Figure 2 


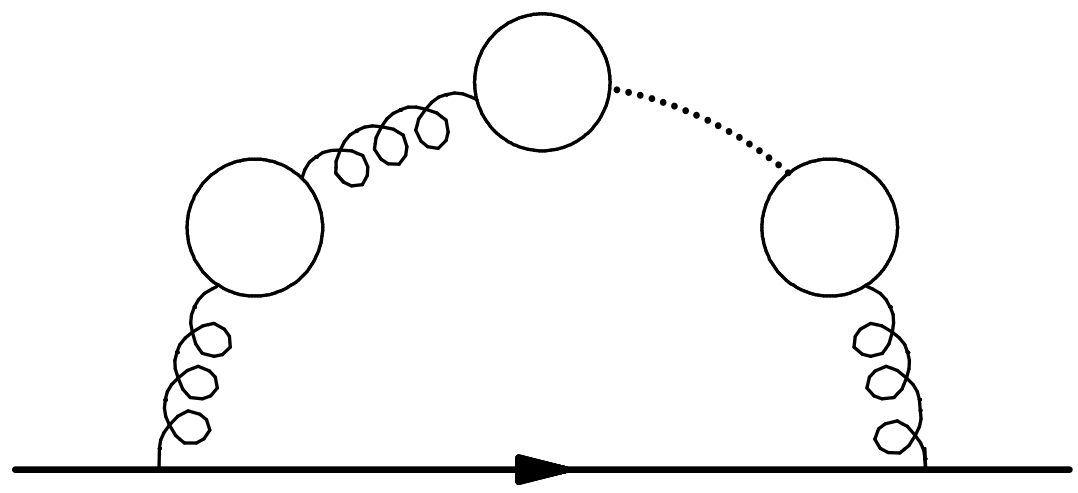

Figure 3

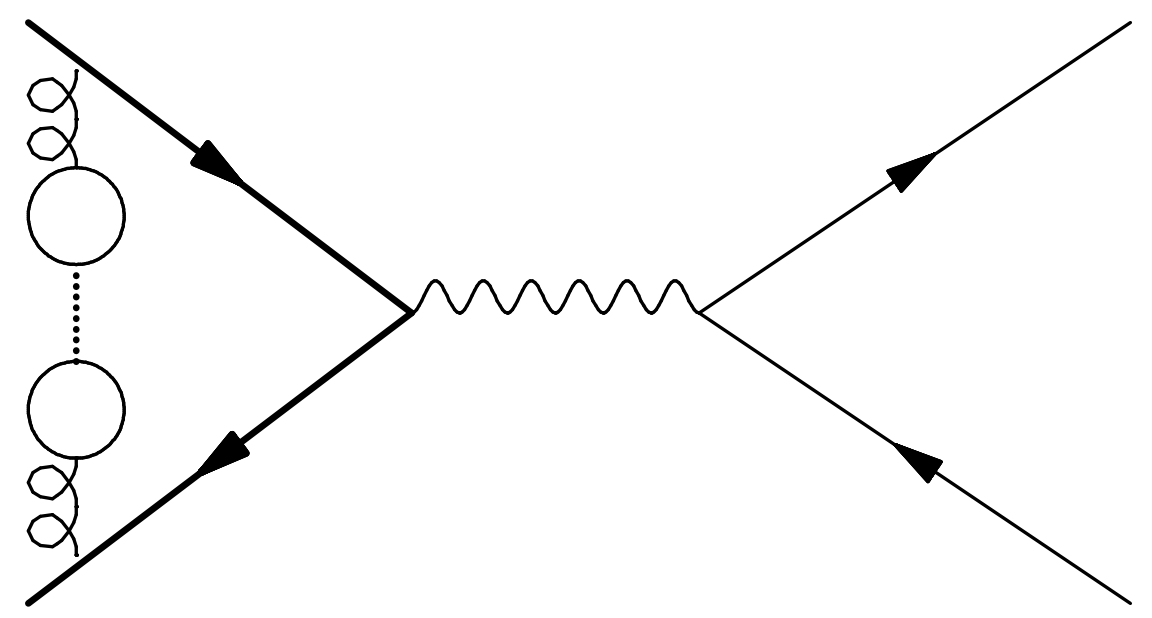

Figure 4 


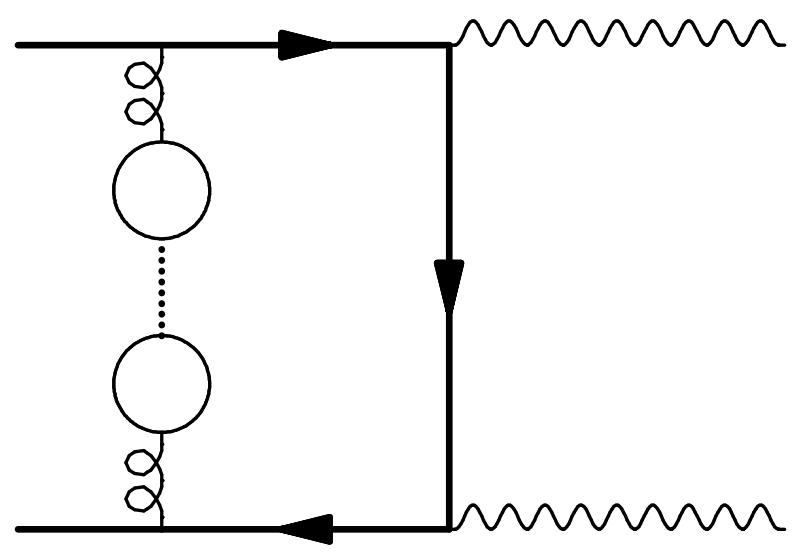

(a)

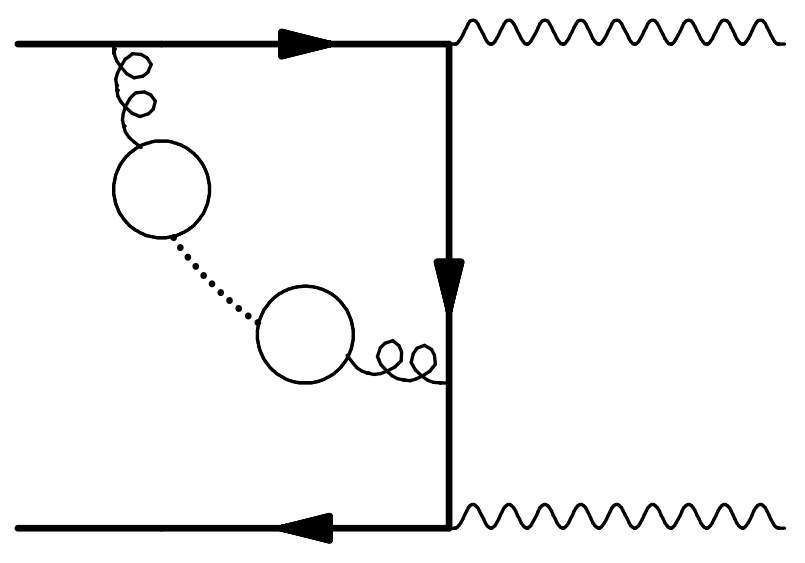

(b)

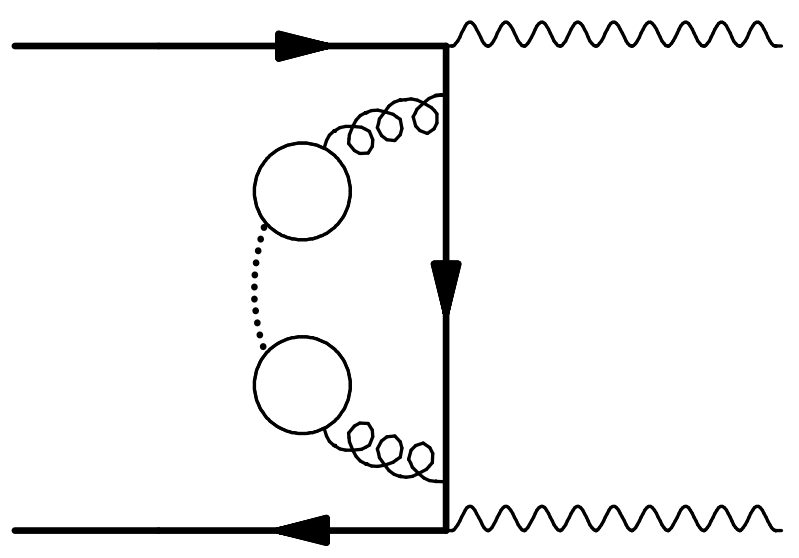

(d)

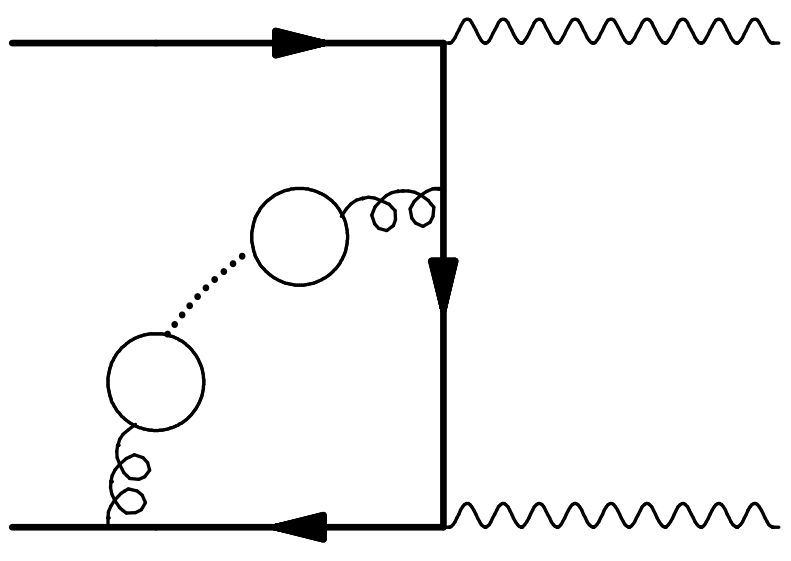

(c)

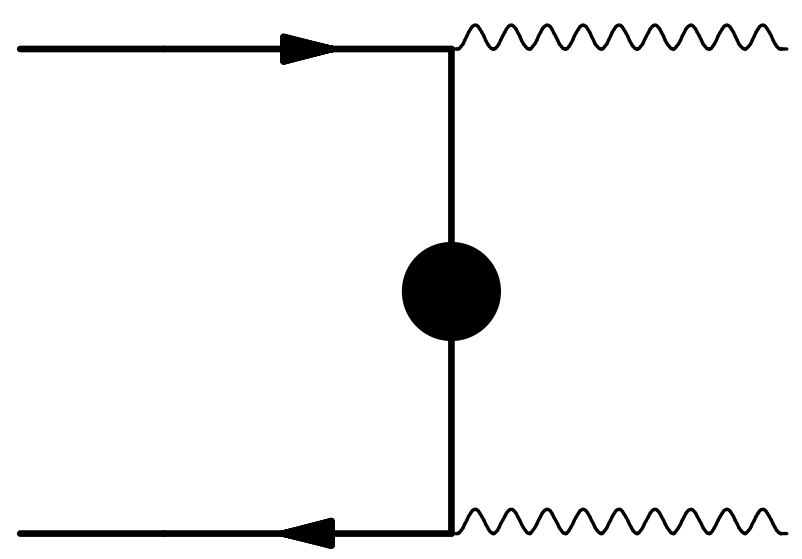

(e)

Figure 5 